Revue des patrimoines

\title{
Les pèlerinages dans les régions dévastées du nord de la France organisés par la Compagnie du chemin de fer du Nord au lendemain de la Première Guerre mondiale
}

\section{Gersende Piernas}

\section{OpenEdition}

\section{Journals}

Electronic version

URL: http://journals.openedition.org/insitu/11420

DOI: $10.4000 /$ insitu. 11420

ISSN: 1630-7305

\section{Publisher}

Ministère de la Culture

Electronic reference

Gersende Piernas, «Les pèlerinages dans les régions dévastées du nord de la France organisés par la Compagnie du chemin de fer du Nord au lendemain de la Première Guerre mondiale », In Situ [Online], 25 | 2014, Online since 10 December 2014, connection on 25 June 2020. URL : http:// journals.openedition.org/insitu/11420; DOI : https://doi.org/10.4000/insitu.11420

This text was automatically generated on 25 June 2020 .

In Situ Revues des patrimoines est mis à disposition selon les termes de la licence Creative Commons Attribution - Pas d'Utilisation Commerciale - Pas de Modification 4.0 International. 


\title{
Les pèlerinages dans les régions dévastées du nord de la France organisés par la Compagnie du chemin de fer du Nord au lendemain de la Première Guerre mondiale
}

\author{
Gersende Piernas
}

1 Jusque-là réservés à quelques érudits lors des conflits précédents et à titre individuel, les pèlerinages se généralisent avec la Grande Guerre. Ils s'effectuent pendant le conflit lui-même par les familles en deuil, en raison des pertes humaines sans précédent sur un territoire très vaste, et aussi par des touristes dès 1917, suite notamment à la publication de Guides Michelin sur la bataille de la Marne traduits en plusieurs langues et à une campagne de communication par affiches.

2 À l'issue du conflit, ces pèlerinages se multiplient et deviennent de véritables remèdes contre ce traumatisme collectif pour les proches des soldats décédés, pour qui un voyage par an est déclaré gratuit en 1921, mais aussi des hommes comme des femmes curieux, des touristes en somme. Des circuits et même des séjours sont organisés, en France et à partir de l'étranger, par des associations, des agences de voyages et des compagnies de chemin de fer, dont les deux plus concernées sont celles de l'Est et du Nord.

3 Et pour cause, au cours de la Première Guerre mondiale, dix départements furent envahis à l'est (Ardennes, Marne, Meurthe-et-Moselle, Meuse, Vosges) et au nord (Nord, Pas-de-Calais, Somme, Aisne, Oise) et subirent des ravages très importants sur les champs de bataille mais aussi dans les villes et les installations industrielles. Le sort de ces régions dévastées du Nord recouvre alors celui de la Compagnie du chemin de fer $\mathrm{du}$ Nord. Ainsi, toutes les installations du chemin de fer comprises entre la ligne 
extrême du front de 1918, et même quelques kilomètres à l'ouest de cette ligne jusqu'à la frontière, ont été complètement détruites dans la majeure partie d'une façon absolument systématique soit $1966 \mathrm{~km}$ de lignes, 350 gares, 1180 ponts, 5 tunnels, dépôts, ateliers, lignes télégraphiques et téléphoniques, électricité, alimentation en eau, signaux. Ces réparations colossales s'élevant à 2500000000 francs $^{1}$, les travaux commencèrent très rapidement si bien qu'une partie du réseau, lignes, gares, ouvrages d'art, furent opérationnels dès le début de l'année 1919.

Compagnie : se relever certes, mais aussi être le vecteur indispensable pour montrer les désastres de cette guerre - à travers ses propres destructions et celles du Nord, les champs de bataille - et pour panser les plaies en permettant aux familles des défunts de s'occuper de leurs morts et disparus. Elle mit alors en place des pèlerinages sur cet espace national traumatisé aussitôt le conflit fini, régénérant alors le tourisme sous une forme nouvelle.

De ce défi, il subsiste deux cartons dans le fonds d'archives de la Compagnie, conservés aux Archives nationales du monde du travail ${ }^{2}$. Composés de correspondances, prospectus, affiches, notes et rapports, et bien que lacunaires, ils permettent, majoritairement sur la période 1919-1921, de retracer la mise en place de ces trains de pèlerinage et leur exploitation.

6 À travers cet article, nous verrons que, entre enjeux politiques, économiques, touristiques et historiques, ces pèlerinages mettent en lumière plusieurs patrimoines mutilés par la guerre (espaces naturels, urbains et industriels) éphémères en cet état mais indispensables à montrer et à figer dans la conscience collective pour dénoncer la barbarie allemande et mettre en valeur l'héroïsme des combattants et des populations.

7 Mais il s'agit également de démontrer que ces trains de pèlerinage sillonnant cette région septentrionale ravagée la sanctifient et l'unissent à la région de l'Est dans un espace mémoriel national, et qu'ils participent à la naissance du mythe du poilu et à la reconnaissance envers les Alliés libérateurs.

8 Pour ce faire, nous aborderons en premier la mise en place de ces trains et leur organisation, puis nous analyserons à travers les informations en provenance de la compagnie et des voyageurs comment on passe du pèlerinage au tourisme de mémoire nationale et à l'imaginaire de la Grande Guerre, et enfin nous traiterons de la commercialisation inévitable de ce tourisme.

\section{Sur les chemins de la Grande Guerre}

9 Lorsque la Compagnie du chemin de fer du Nord instaure en 1919 des trains de pèlerinages dans les régions dévastées, elle puise dans son expérience des trains de pèlerinages religieux au cours $\mathrm{du}$ XIX $\mathrm{x}^{\mathrm{e}}$ siècle, siècle de la renaissance des pèlerinages mariaux, tout comme dans son expérience des trains spéciaux mis en place à l'occasion de manifestations extraordinaires parisiennes ou régionales (expositions, rencontres sportives, foires commerciales, etc.). Elle s'inspire également d'un projet datant du début de l'année 1918 auquel elle a pris part.

Dans le contexte de la reconstruction, le 28 février 1918, une commission d'études rassemble autour du directeur de l'office national du tourisme, un ingénieur des Ponts et Chaussées, les compagnies du chemin de fer de l'Est, du Nord, du PLM, la Société générale de transport automobile et la commission interministérielle des Souvenirs de 
guerre ${ }^{3}$. Il s'agit d'une prise de contact pour mettre sur pied l'organisation qui pourrait être créée en vue de la visite des souvenirs de guerre dont la liste a été dressée par la mission du général Vidalon pour être classés comme souvenirs historiques afin d'éviter qu'ils soient détruits. La visite de ces souvenirs historiques, répartis sur tout l'ensemble $\mathrm{du}$ front, comporte, sur proposition du ministère de la Guerre, 10 secteurs dans la zone des Armées. Ces secteurs seraient desservis, au départ de certains centres importants atteints par le chemin de fer, au moyen de voitures automobiles qu'une société anonyme mettrait en service et exploiterait à partir de ces centres, soit une tournée journalière de 4000 kilomètres. En ce qui concerne le réseau du Nord, les secteurs envisagés seraient les suivants :

- Soissonnais, avec Soissons comme point de départ,

- Noyonnais, avec Noyon comme point de départ,

- Artois, avec Albert, Arras comme points de départ,

- Yser, avec Hazebrouck comme point de départ.

11 Ces circuits touristiques dans les régions dévastées en train et prolongés en automobile, seraient à tarifs uniques avec des billets communs et une publicité générale. Le ministère de la Guerre louerait 100 châssis de voitures militaires parmi les meilleurs de ceux qui resteraient après la Guerre, comme une sorte de subvention de l'État. Sur ces châssis seraient montées des carrosseries. Une société exploitante serait formée, pour une durée de 5 ans, au capital de 1500000 francs. Un intérêt de $7 \%$ serait garanti aux actionnaires par un consortium formé des compagnies de chemins de fer intéressées. Mais le projet échoue faute de participations financières suffisantes des protagonistes, bien que cette "affaire para[isse] extrêmement intéressante " et qu'elle "doit être prospère [...] et qu'il y aurait matière à un trafic intéressant d'après guerre " selon la Compagnie du Nord. Il échoue aussi parce que le réseau des compagnies ferroviaires les plus impactées n'est pas encore prêt.

Pourtant, dès la remise en état d'une partie des rails du réseau du Nord et la reprise du trafic, des trains spéciaux sont organisés à partir de février 1919 pour les représentants de la presse étrangère accrédités à la conférence de la Paix. Cette dernière prône la mise en place de tels voyages car ils sont susceptibles d'exercer une grande influence sur l'opinion qui doit se faire jour en France comme à l'étranger de la situation exceptionnelle faite à la France, parmi tous les États belligérants, par les ravages qu'elle a subis.

Après les journalistes, sont aussi conviés en mars et avril 1919 les représentants des chambres de commerce françaises et étrangères, accompagnés des industriels concernés, afin de découvrir toutes ces destructions, les évaluer et de projeter leur reconstitution, comme c'est le cas des lignes de chemin de fer et des compagnies minières (fig. 1). 
Figure 1

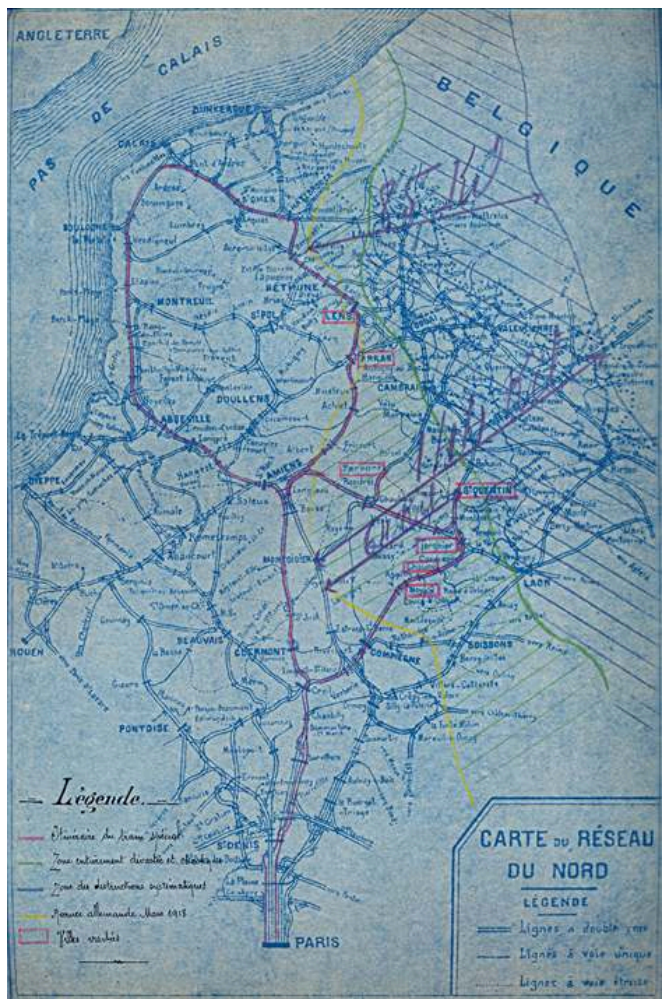

Carte du trajet du train spécial pour la visite des présidents des chambres de commerce de France avec mention de la zone de front et des destructions, [mars-avril 1919]. Archives nationales du monde du travail, fonds de la Compagnie du chemin de fer du Nord, 202 AQ 1638.

Phot. Piernas, Gersende. (C) Archives nationales du monde du travail.

14 Ce sont donc ces premiers témoignages qui encouragent la Compagnie du chemin de fer $\mathrm{du}$ Nord à instaurer quelques mois plus tard les trains de pèlerinages. Le ministère des Travaux publics et des Transports approuve les propositions de la Compagnie de mettre en marche, à partir du 11 mai 1919 les dimanches, un train d'excursion Paris-AlbertArras-Lens-Paris qui traverse les champs de bataille de la Somme et de l'Artois.

Une circulaire avec tous les détails techniques est envoyée au réseau. Il part de Paris à

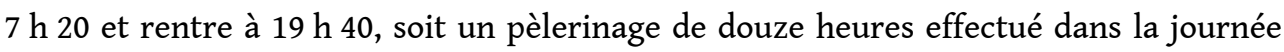
(fig. 2). S'ajoute un prospectus-horaires destiné au public édité aussi en anglais, qui met en plus en garde contre les dangers de la zone visitée, notamment à cause des débris et des munitions subsistants. 
Figure 2

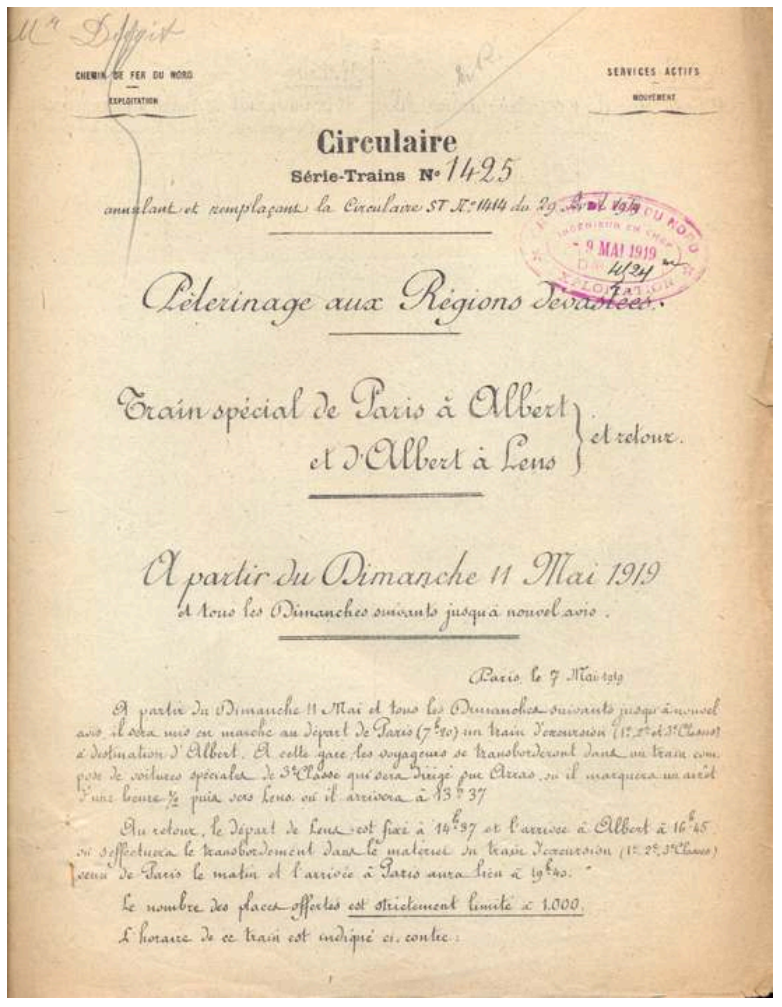

Circulaire $n^{\circ} 1425$ du Service exploitation de la Compagnie du chemin du Nord sur les modalités techniques de la mise en place le 11 mai 1919 du premier train de pèlerinage, 7 mai 1919. Archives nationales du monde du travail, fonds de la Compagnie du chemin de fer du Nord, 202 AQ 1638.

Phot. Piernas, Gersende. (c) Archives nationales du monde du travail.

Le train est pris à marche rapide jusqu'à Albert, puis les voyageurs empruntent des baladeuses, des voitures-tramways transformées dans les ateliers de la gare de La Chapelle en sorte de wagons découverts spécialement aménagés. Il comporte 1000 places réparties en 3 classes, dont le prix du billet est compris entre $23,40 \mathrm{~F}$ et $42,80 \mathrm{~F}$ avec la possibilité de réserver pour $1 \mathrm{~F}$ de plus.

17 Ces trains spéciaux, d'abord dominicaux, circulent, quelque temps après aussi le jeudi (fig. 3). 
Figure 3

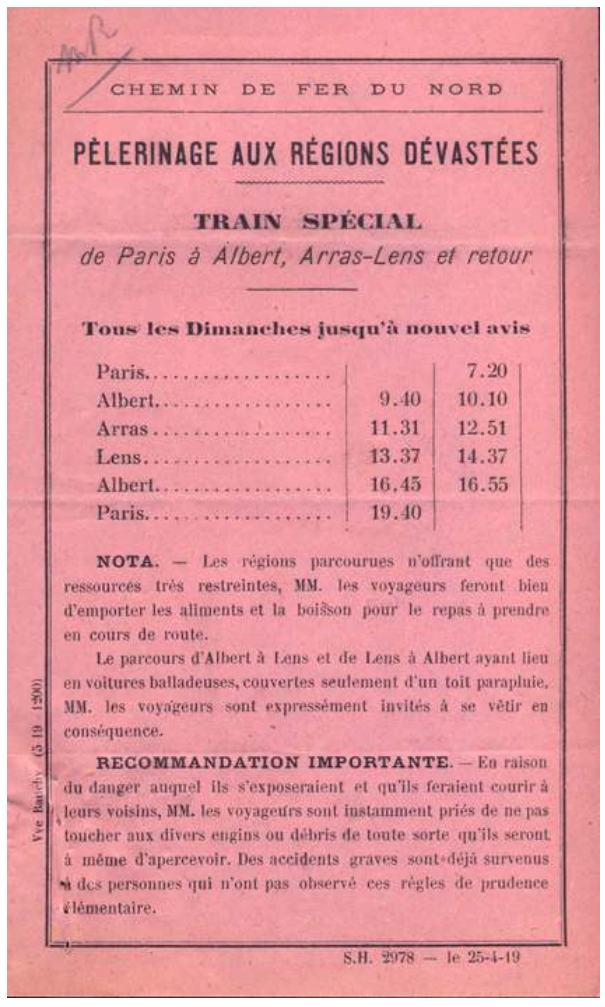

Prospectus-horaires du premier train de pèlerinage dans les régions dévastées circulant à partir du 11 mai 1919 sur le Réseau du Nord, [avril-mai 1919]. Archives nationales du monde du travail, fonds de la Compagnie du chemin de fer du Nord, 202 AQ 1638.

Phot. Piernas, Gersende. (C) Archives nationales du monde du travail. par des prospectus en français comme en anglais :

- à partir du 15 juin, le $2^{\mathrm{e}}$ pèlerinage intitulé « Bataille de la Somme » circule les dimanches et les mardis, puis à partir du 9 octobre plus que les dimanches. Ils parcourent aller-retour Paris, Montdidier, Chaulnes, Péronne, Cléry, Maurepas en dix heures. Le billet coûte entre 18 et $35 \mathrm{~F}$.

-à partir du 13 juin 1919, le $3^{e}$ pèlerinage intitulé "Chemin des Dames » circule les dimanches, jeudis et fête. L'aller-retour, Paris, Coucy, Anizy, Chailvet, Chemin des Dames, Crouy, Soissons, prend douze heures et coûte entre 35 et $51 \mathrm{~F}$. À partir de Coucy, le circuit s'effectue avec les autobus de la Société de construction et d'entretien de matériel industriel et agricole (SCEMIA) avec qui une convention est passée.

- à partir de juillet 1919, le $4^{\mathrm{e}}$ pèlerinage intitulé « Une journée aux champs de bataille francoanglais » circule tous les jours. Le circuit, Paris, vers Albert ou Arras, ou Albert vers Arras, via Bapaume, Bullecourt, Vimy et Lens, s'effectue en quinze heures. Le billet coûte entre 85 et $99 \mathrm{~F}$, avec seulement deux classes ( $1^{\mathrm{e}}$ et $2^{\mathrm{e}}$ classes). Le circuit est combiné avec les automails ${ }^{4}$ de la Société française des auto-mails patronnée par l'office national du tourisme. Cette société a proposé en juin 1919 ces combinaisons car elle jouit d'une situation privilégiée. Elle possède un matériel neuf, 20 grands autocars commandés quelques jours après l'Armistice et qui permettent de s'affranchir du tracé ferroviaire et de ses destructions en s'approchant très près des lieux à visiter. En août 1919, il est possible d'admettre des voyageurs de province qui gagneraient Arras ou Albert sans passer par Paris et pour lesquels les auto-mails vendraient des billets afférents au parcours automobile seulement. 
-à partir du 25 octobre 1919, le 5e pèlerinage intitulé "Les Champs de bataille d'Ypres ", parcourt tous les jours en quatorze heures Paris, Lille, Armentières, Locre, Ypres, Gheluvelt. Le billet coûte entre 93 et $110 \mathrm{~F}$, avec seulement deux classes ( $1^{\mathrm{e}}$ et $2^{\mathrm{e}}$ classes). deux partenaires, mais aussi auprès de l'office national du tourisme et des agences de voyages françaises et étrangères. Les guides-accompagnateurs des sociétés SCEMIA et Auto-mails, tout comme les familles des agents de la Compagnie du Nord, peuvent monter gratuitement à bord. Les accompagnateurs des agences de voyage circulent gratuitement avec des groupes d'au moins 20 personnes. De plus, des réductions peuvent être accordées sur demande et beaucoup de places sont offertes à des officiels.

21 À côté de ces circuits proposés sont mis en place des trains spéciaux sur demande, à partir de 1000 personnes. Des billets aux tarifs calculés sont émis pour ces ventes à guichet fermé (fig. 4). Ces trains spéciaux peuvent comporter des wagons-lits et des wagons-restaurants en plus des baladeuses.

\section{Figure 4}

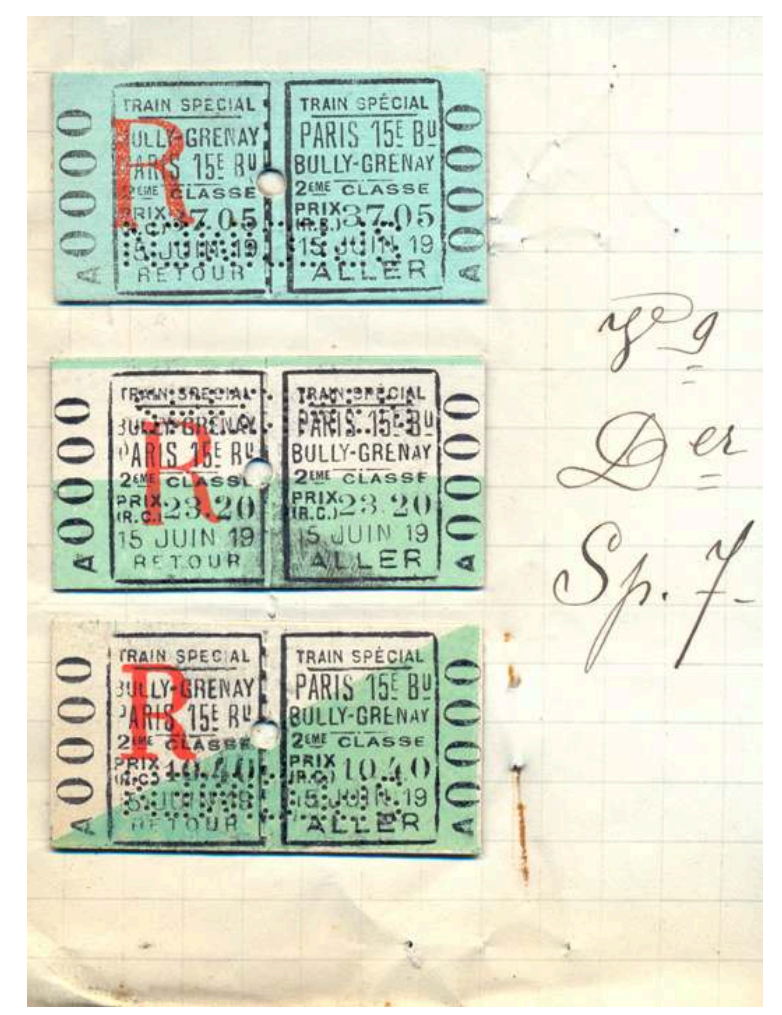

Billets du train spécial de Paris à Arras-Lens-Bully-Grenay circulant le 15 juin 1919 et réservé par la Société des anciens élèves de l'École polytechnique de Paris. Archives nationales du monde du travail, fonds de la Compagnie du chemin de fer du Nord, [15 juin 1919]. Archives nationales du monde du travail, fonds de la Compagnie du chemin de fer du Nord, 202 AQ 1638.

Phot. Piernas, Gersende. (C) Archives nationales du monde du travail.

Par ailleurs, puisque ces circuits et trains spéciaux rencontrent un franc succès en France et à l'étranger, il est même émis le projet de les raccorder à ceux du réseau Est et $d u$ réseau belge lors d'une conférence internationale entre les représentants des 
compagnies de chemin de fer de Belgique, Angleterre, Pays-Bas et France qui s'est tenue les 22 et 23 avril 1920 au siège de la Compagnie du chemin de fer du Nord. La Great Eastern Railway et les Chemins de fer néerlandais proposent des billets circulaires entre les champs de bataille de France et de Belgique, projet qui, en raison de difficultés financières et techniques, ne verra pas le jour (fig. 5).

\section{Figure 5}

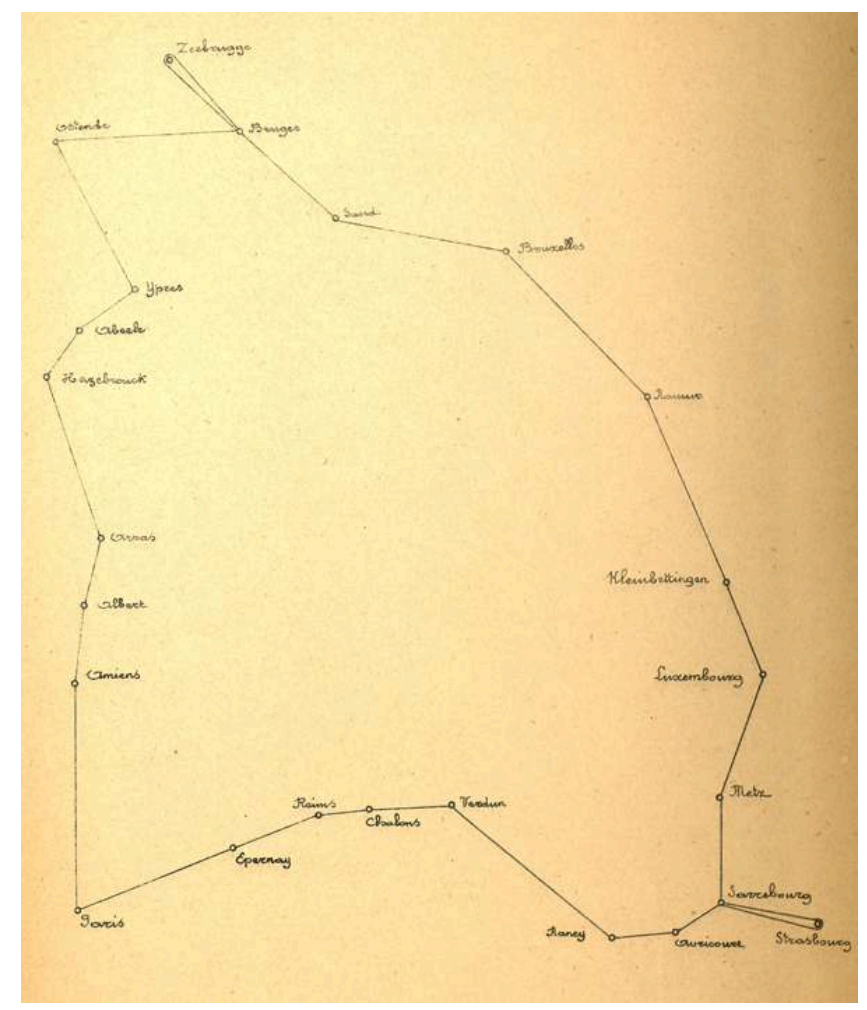

Circuit $n^{\circ} 2$ du pèlerinage commun entre le Nord, l'Est et la Belgique, prévu dans le procès-verbal de la conférence internationale entre les représentants des compagnies de chemin de fer de Belgique, Angleterre, Pays-Bas et France qui s'est tenue les 22 et 23 avril 1920 au siège de la Compagnie du chemin de fer du Nord, [avril 1920]. Archives nationales du monde du travail, fonds de la Compagnie du chemin de fer du Nord, 202 AQ 1639.

Phot. Piernas, Gersende. (c) Archives nationales du monde du travail.

Faute de fréquentation suffisante, de l'arrivée de l'hiver, de la baisse des jours, de l'état des routes avec chaussée parfois en bois obligeant le changement régulier des pneumatiques, denrées encore rares et coûteuses, ou bien l'emprunt de la route nationale plus rapide, les circuits de la Compagnie du chemin de fer du Nord cessent à partir du 17 novembre 1919 pour reprendre l'année suivante en juin. Au total, on compte pour l'année 1919, 51585 voyageurs et une recette de 1352 460,25 F. Le nombre de voyageurs varie entre quelques centaines et 8000 voyageurs par mois, soit quand même dix fois moins que pour les trains de pèlerinages religieux avant 1914 et cinq fois plus que le pèlerinage à Lourdes 5 .

Les circuits Paris-Lens et Paris-Coucy-le-Château demeurent les plus prisés.

Les chiffres de l'année suivante sont moindres si bien que la Compagnie du Nord annonce ne pas s'engager pour 1921, ce qui semblerait être attesté par l'absence de dossier dans le fonds. Seule l'année 1926 voit une demande pour un train de pèlerinage 
organisé par le Comité commémoratif de la Somme à partir de Paris à destination des champs de bataille de la Somme et combiné avec des autos et des auto-camions (fig. 6).

Figure 6

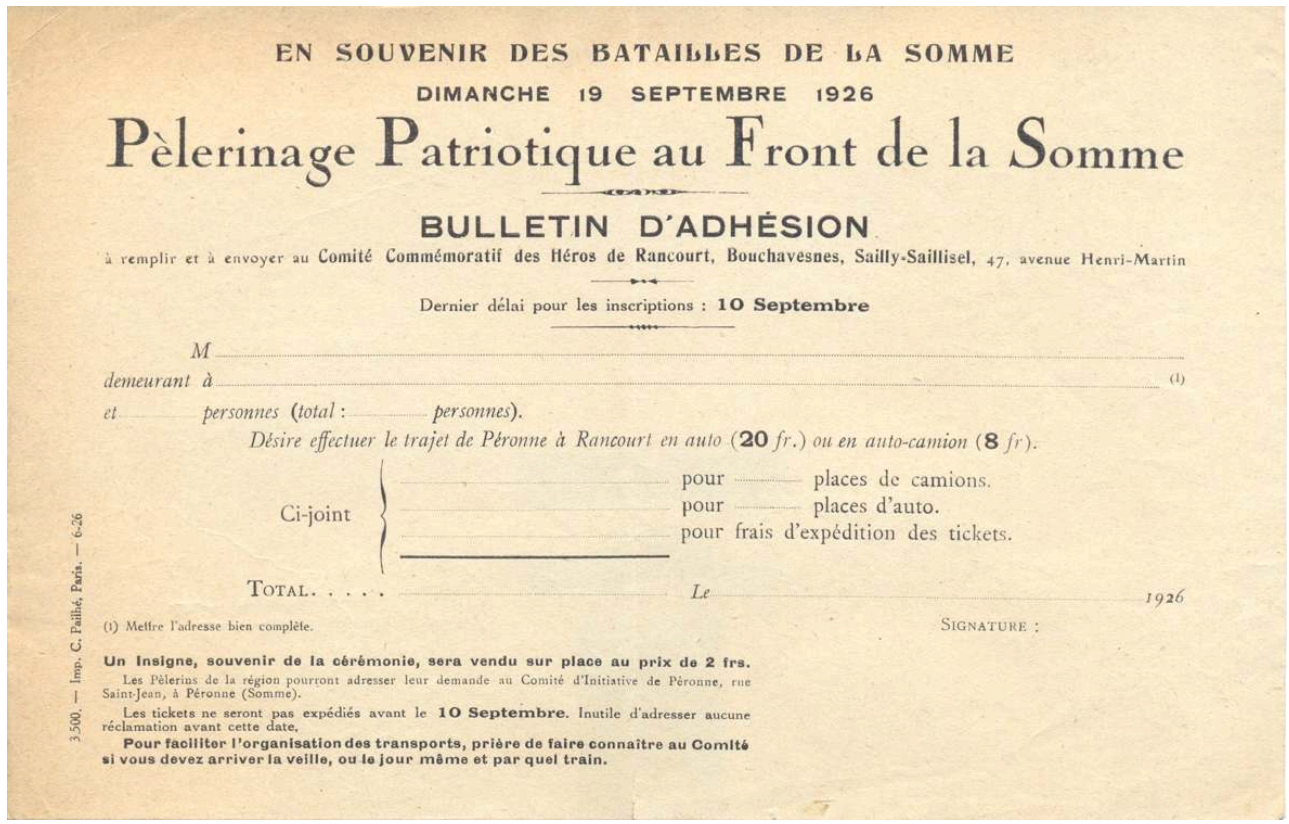

Bulletin d'adhésion au pèlerinage sur les champs de bataille de la Somme organisé par le Comité commémoratif de la Somme le dimanche 19 septembre 1926, [septembre 1926]. Archives nationales du monde du travail, fonds de la Compagnie du chemin de fer du Nord, 202 AQ 1638.

Phot. Piernas, Gersende. (c) Archives nationales du monde du travail.

Du peu de ce qui nous est parvenu de ces deux ans d'exploitation, nous pouvons néanmoins donner les contours de la teneur de ces pèlerinages, de la clientèle et de leur retentissement.

\section{Du pèlerinage du Souvenir au tourisme de mémoire nationale et à l'imaginaire de la Grande Guerre}

L'objectif de la Compagnie du chemin de fer du Nord est clairement édicté sur ses prospectus-horaires. Elle souhaite montrer les ravages commis par les Allemands et l'ampleur des offensives victorieuses des Français et des Anglais. Le choix des circuits respecte à la fois les différentes zones de batailles (Somme, Artois, Chemin des Dames, etc.) et la zone d'occupation, mais aussi le maillage de la Compagnie, qui, insuffisant pour atteindre certains endroits, s'allie alors au transport routier. Ces pèlerinages sont donc assurément le seul vrai moyen de montrer au public la désolation profonde des régions dévastées en y pénétrant intimement. C'est aussi un vecteur puissant de propagande pour faire garder le souvenir des crimes de l'ennemi et des souffrances endurées par les Français.

De ces pèlerinages, nous gardons de nombreux récits conservés dans les archives de la Compagnie du chemin de fer du Nord. Les premiers remontent à février 1919 et concernent les circuits réalisés, aussitôt la reprise du trafic, pour les représentants de la presse étrangère accrédités à la conférence de la Paix. Ils prennent la forme de 
lettres envoyées à la Compagnie, accompagnées d'articles publiés dans la presse française ou étrangère (anglaise et américaine).

Ces journalistes expriment tous leur indignation contre le peuple allemand et leur vive émotion parfois jusqu'aux larmes car ils ont pris ces trains - réinstaurés depuis quelques jours bien avant les premiers circuits de pèlerinages - qui circulent entre ruines, tranchées, sapes et tombes parfois. Le danger est partout avec les débris, les dénivellations, les barbelés et les munitions. Tout n'est que ruines, destructions et désolation. Le journaliste George $C$. Curnock raconte dans son article intitulé « Lens as it is ", publié le 25 février 1919 dans le Daily Mail, comment il fait la connaissance des habitants d'Arras obligés de vivre dans leurs caves. Oliver Owen Kuhn indique en février 1919 qu'il n'y avait dans ces destructions complètes aucune nécessité militaire mais seulement un "esprit de vengeance et de lucre». Pour lui, il faut "forcer l'Allemagne à sentir le fardeau de sa honte parmi les peuples civilisés, il faut qu'elle paye et avant tout qu'elle paye complètement $»^{6}$.

Ces récits ou articles peuvent même être accompagnés de photographies. Beaucoup ont été prises en tant que souvenirs et comme preuves pour les besoins des réparations et des indemnités. Si beaucoup ont été envoyées à la Compagnie du chemin de fer du Nord, seules quelques-unes subsistent dans son fonds d'archives, comme celles prises lors du pèlerinage fin mars 1919 des représentants des chambres de commerce de France et ayant trait aux destructions de la ligne Arras-Lens (fig. 7).

Figure 7

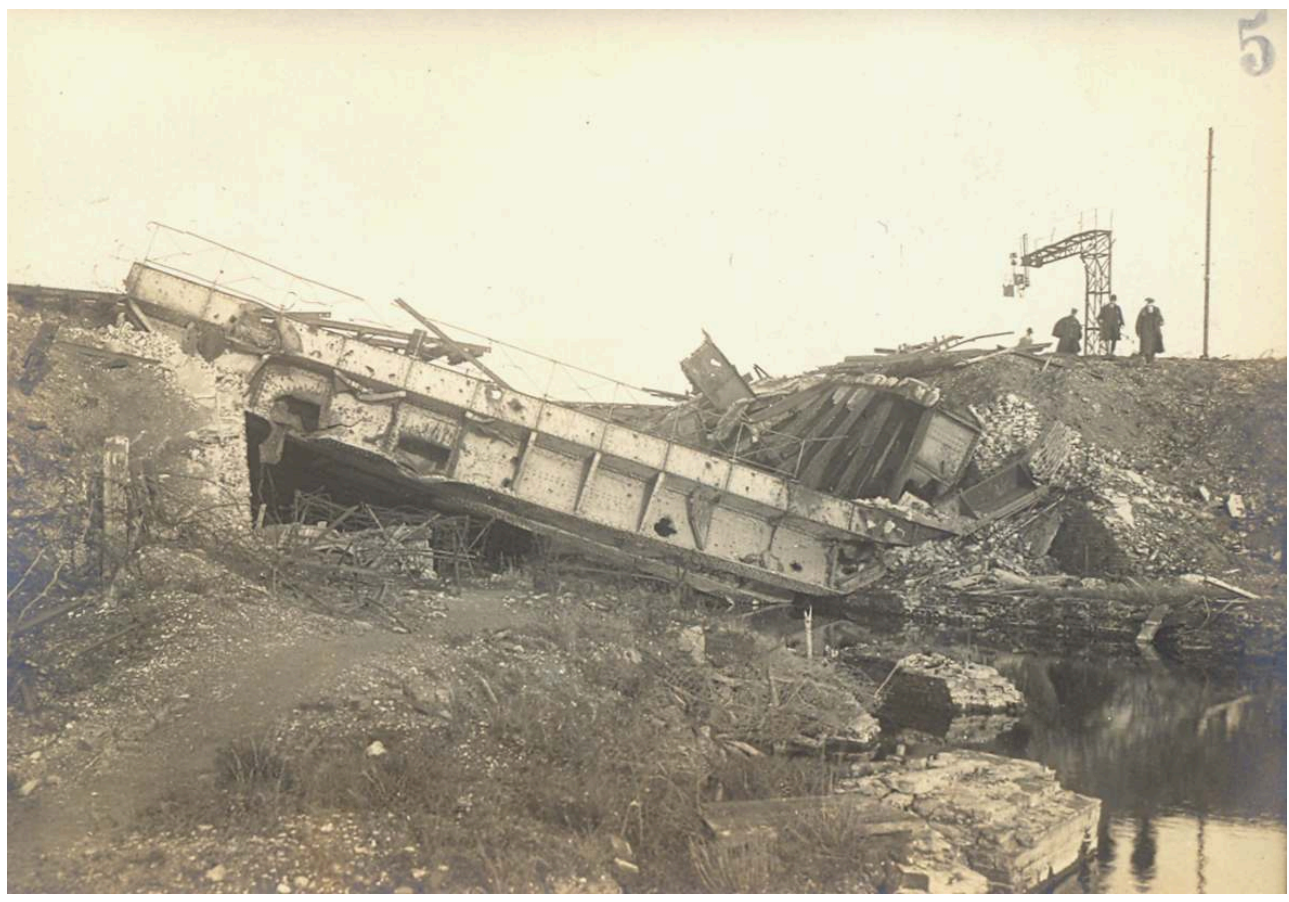

Photographie des destructions du pont de chemin de fer sur la Deûle à Lens (ligne Arras-Lens) prise lors de la visite des présidents des chambres de commerce de France, [mars-avril 1919]. Archives nationales du monde du travail, fonds de la Compagnie du chemin de fer du Nord, 202 AQ 1638.

Phot. Piernas, Gersende. (c) Archives nationales du monde du travail.

31 Tous ces récits assurent donc une publicité dans la presse et suscitent un intérêt notoire du grand public. C'est pourquoi, afin d'orienter au mieux le pèlerin, des guides les accompagnent, soit en provenance de leur agence de voyage, soit affrétés par la 
Compagnie du chemin de fer du Nord ou des sociétés d'autobus. Il s'agit d'ailleurs le plus souvent de contrôleurs ou conducteurs qui tirent leurs connaissances des brochures vendues par la compagnie à bord des trains et en gare, sur le modèle des Guides Michelin.

Le voyage du premier train de pèlerinage, durant une journée, est donc couvert largement par la presse (fig. 8, 9). Il s'agit de familles de soldats et surtout de curieux, des hommes, des femmes et des enfants.

Figure 8

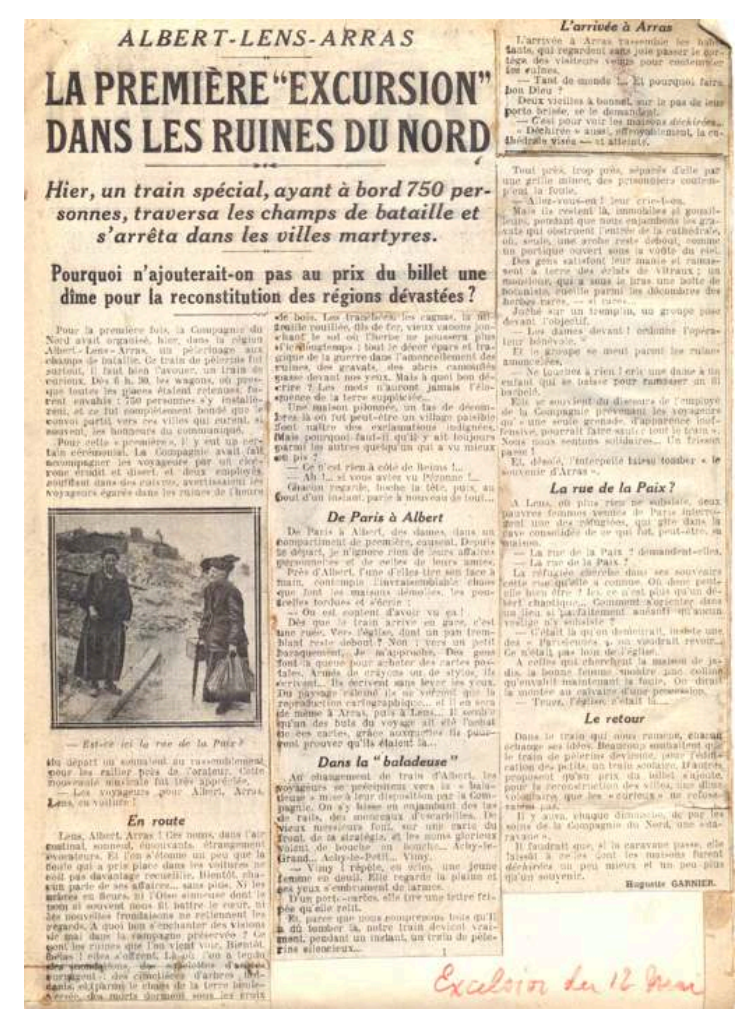

Article d'Huguette Garnier sur le premier train de pèlerinage du 11 mai 1919, Excelsior, [12 mai 1919]. Archives nationales du monde du travail, fonds de la Compagnie du chemin de fer du Nord, $202 \mathrm{AQ} 1638$.

Phot. Piernas, Gersende. (c) Archives nationales du monde du travail. 


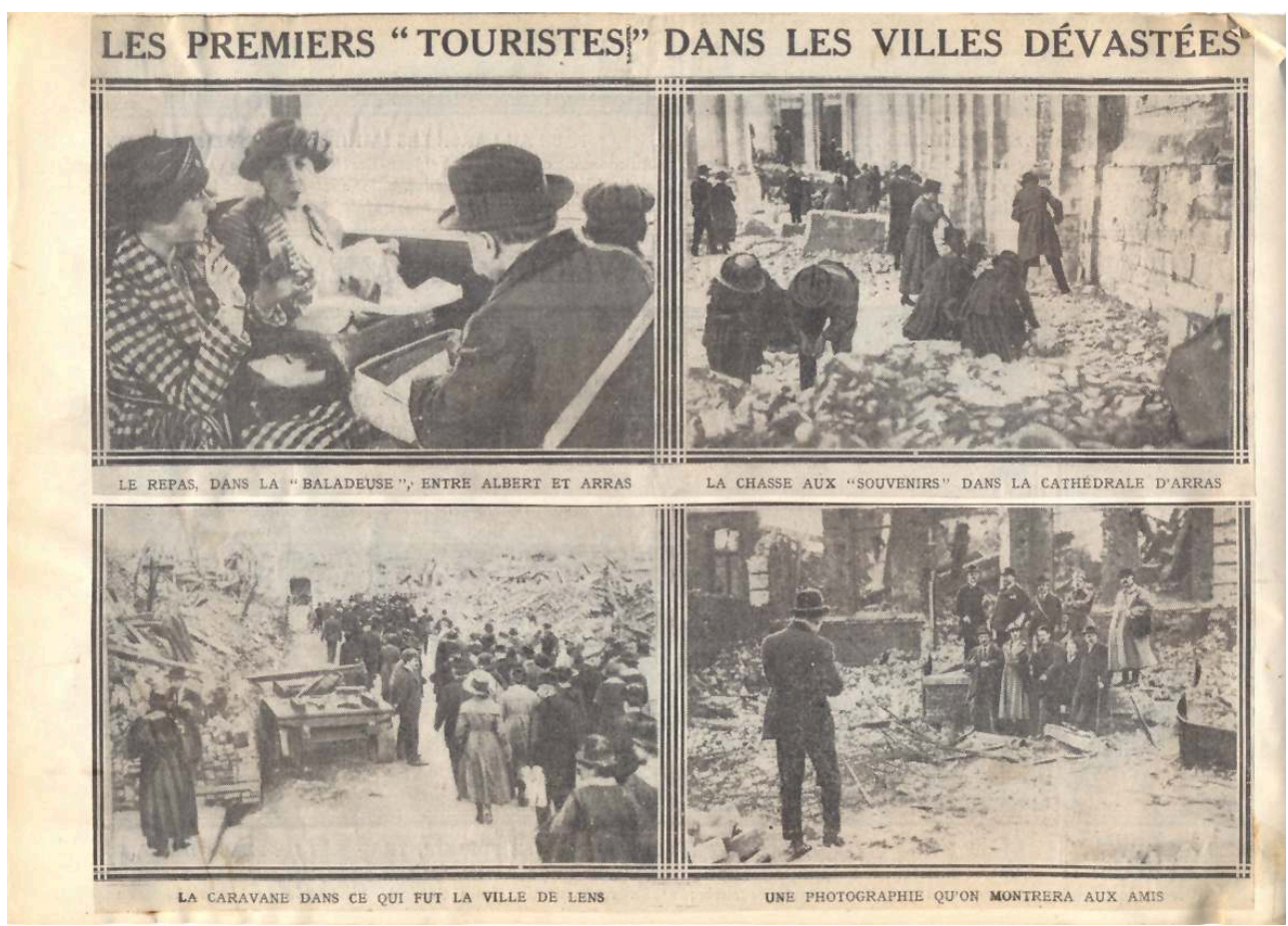

Photographies d'un article sur le premier train de pèlerinage du 11 mai 1919, [Excelsior], [12 mai 1919]. Archives nationales du monde du travail, fonds de la Compagnie du chemin de fer du Nord, 202 AQ 1638

Phot. Piernas, Gersende. (c) Archives nationales du monde du travail.

L'ambiance pendant le trajet en train est quant à elle bien décrite dans la presse, les voyageurs discutent de leurs affaires puis, lors du défilé devant les ruines, c'est la stupeur, la comparaison obligée avec d'autres sites visités juste avant et la remémoration d'un proche tombé. Les visites s'accompagnent de prises de souvenirs, débris, objets, etc., sortes de reliques. On ne manque pas de se faire photographier en souvenir devant le spectacle. Le circuit dans les ruines se fait au clairon des guides avec plus ou moins de surveillance quant aux risques immédiats. On y croise des habitants mais aussi des prisonniers allemands, qui aident à la reconstruction, et qui font les frais de la haine des visiteurs à leur passage.

En outre, les notes internes et les nombreuses demandes de réservation, de réduction, de gratuité ${ }^{7}$ voire d'organisation de trains spéciaux, nous donnent une idée de l'identité de ces pèlerins.

En dehors des officiels et des invités de marque, comme le prince Karapoutra de Siam avec sa suite qui demande un train spécial pour aller de Paris à Lens en août 1920, on retrouve principalement des écoles et des associations du monde de l'instruction (enseignement ?), dont la majorité vient de Paris et d'île-de-France, et quelques-unes des régions de la Loire et de la Bourgogne. Pour elles, il s'agit d'organiser, à l'attention des enfants, des voyages scolaires ou des vacances dans un but patriotique et d'éducation morale (fig. 10). Certains voyages sont même prévus pour les élèves les plus méritants, comme une récompense suprême. C'est pour eux que la Compagnie fait circuler ces trains le jeudi aussi, jour où ils n'ont pas classe. 
Figure 10

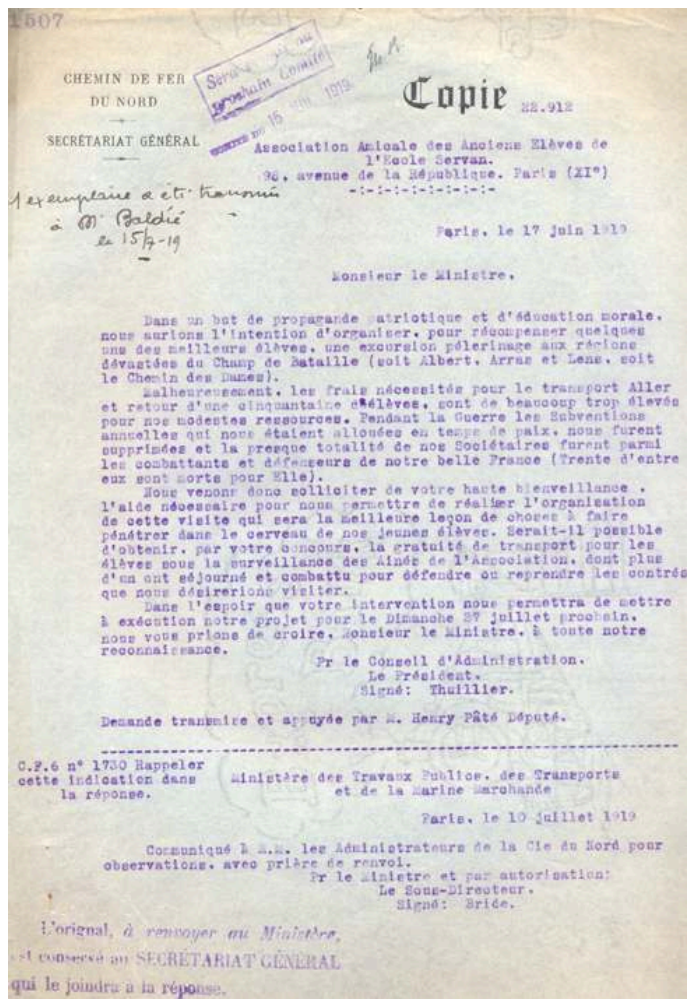

Copie de la demande de réservation et de gratuité pour 50 élèves envoyée par l'Association amicale des anciens élèves de l'École de Servan (Paris), 17 juin 1919. Archives nationales du monde du travail, fonds de la Compagnie du chemin de fer du Nord, 202 AQ 1638.

Phot. Piernas, Gersende. (C) Archives nationales du monde du travail.

Ces pèlerinages sont donc conçus et perçus comme un procédé pédagogique efficace : "C'est le meilleur moyen de frapper l'esprit de ces enfants ${ }^{8}$ ». Au cours de ces pèlerinages devant servir de leçon de patriotisme et servir d'hommage aux héros morts pour la France, des initiatives collectives sont prises. Certains élèves disposent spontanément des bouquets de fleurs sur des tombes et font des dons aux écoliers rencontrés et dépourvus d'école. Les instituteurs en font le récit une fois rentrés dans le bulletin de leur établissement ou de leur association. Ils décrivent les paysages naturels, industriels et urbains dévastés et mesurent ainsi les dégâts par rapport à la situation d'avant-guerre afin de mieux mettre en relief la barbarie allemande (fig. 11). Les champs de bataille ont donc une vocation pédagogique et sont de véritables cours d'histoire grandeur nature. 
Figure 11 a et $b$

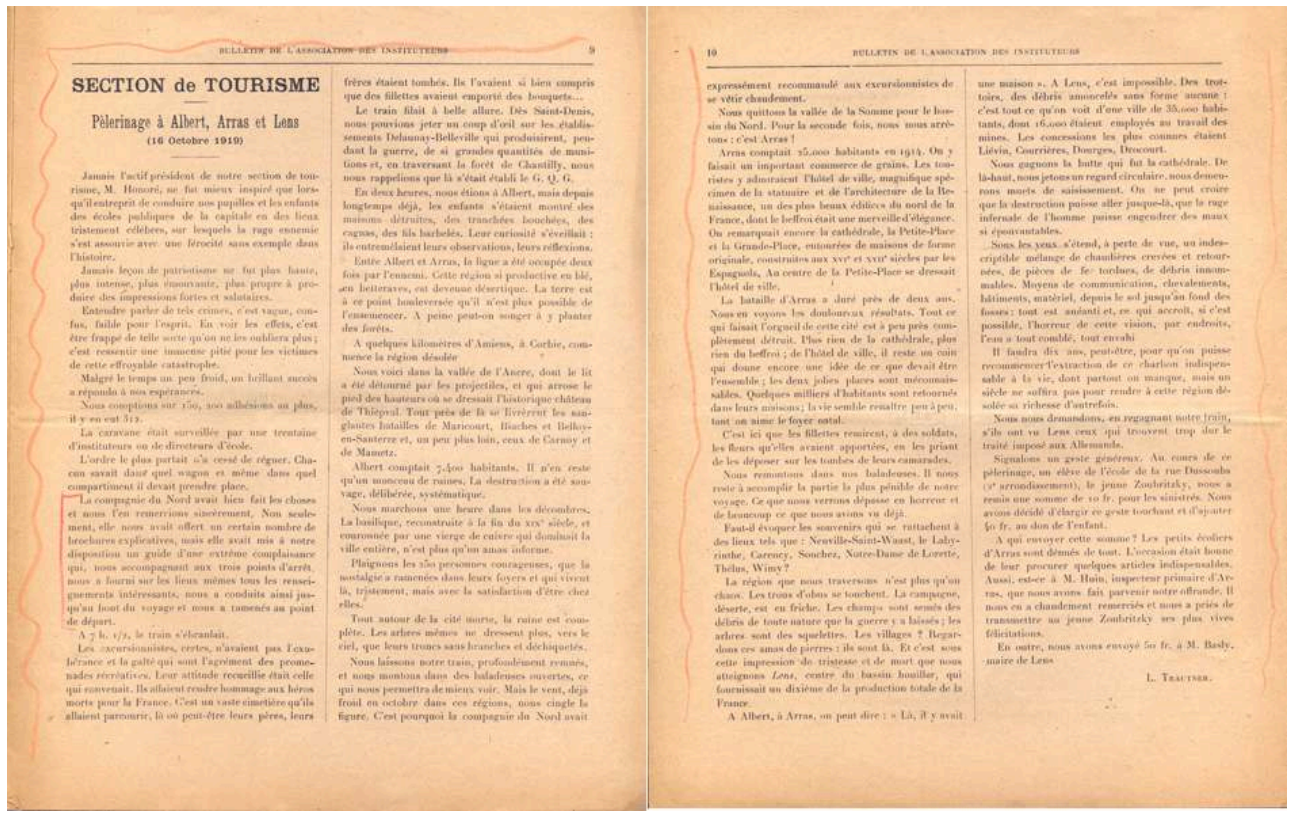

Article de $\mathrm{L}$. Trautner sur le pèlerinage de la Section de tourisme de l'Association des instituteurs le 16 octobre 1919 à Albert, Arras et Lens, publié dans le Bulletin de l'Association des instituteurs, 28 ème année, $n^{\circ} 357$, novembre-décembre 1919-janvier 1920, p. 8-9. Archives nationales du monde du travail, fonds de la Compagnie du chemin de fer du Nord, 202 AQ 1638.

Phot. Piernas, Gersende. (c) Archives nationales du monde du travail.

Des excursionnistes anglais, belges, suisses et américains - bien qu'ayant des difficultés de transport par manque de navires entre l'Amérique et la France - réservent par l'intermédiaire d'agences de voyage aussi dans ces trains. Ils cherchent en premier lieu à découvrir les ruines industrielles mais également, comme beaucoup de familles françaises, à se rendre là où leurs proches se sont battus, sont morts ou ont été portés disparus, à l'instar de nombreuses associations françaises qui organisent la visite des tombes, ce qui est le cas de l'Association du Diable bleu en 1920. D'autres, comme plus tard en 1926, organisent des pèlerinages autour de monuments commémoratifs pour se souvenir, tel le Comité commémoratif de la Somme, dont la présidente n'est autre que Mme la maréchale Foch (fig. 12, 13). Cette dernière mention de pèlerinage dans les archives de la Compagnie du Nord annonce déjà le temps des monuments aux morts en cours de construction, des recueillements collectifs, locaux, régionaux et nationaux : le temps de la commémoration loin du spectacle des ruines. 
Figure 12

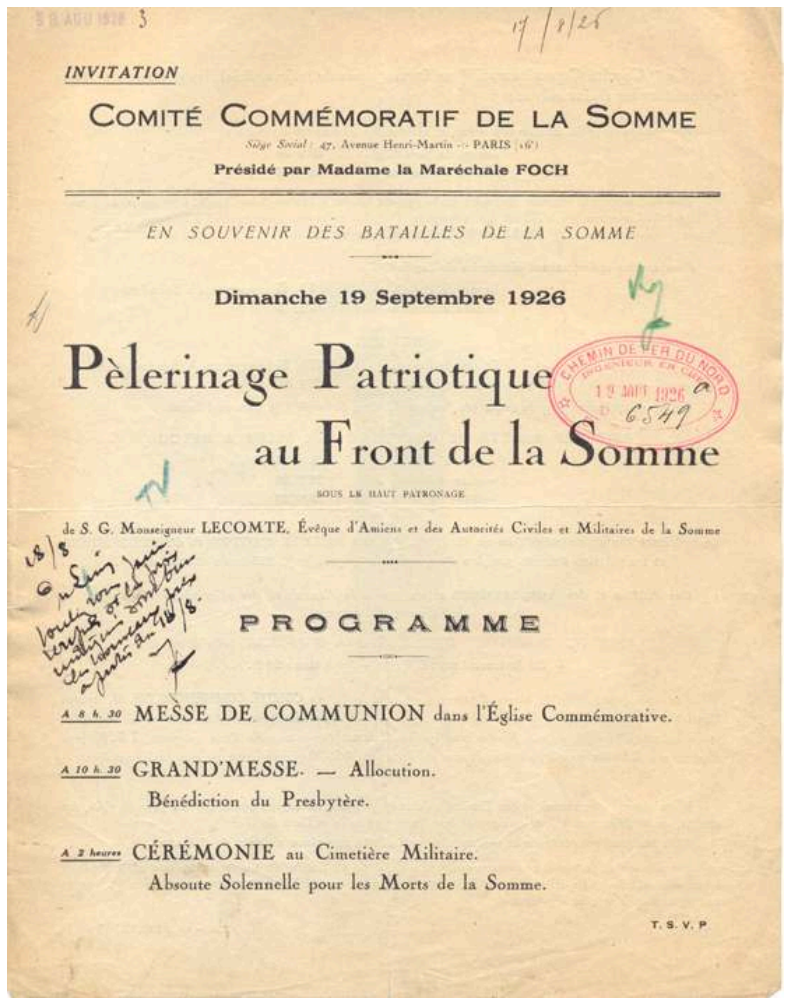

Programme du pèlerinage sur les champs de bataille de la Somme organisé par le Comité commémoratif de la Somme le dimanche 19 septembre 1926, [septembre 1926]. Archives nationales du monde du travail, fonds de la Compagnie du chemin de fer du Nord, 202 AQ 1638.

Phot. Piernas, Gersende. (c) Archives nationales du monde du travail.

\section{Figure 13}

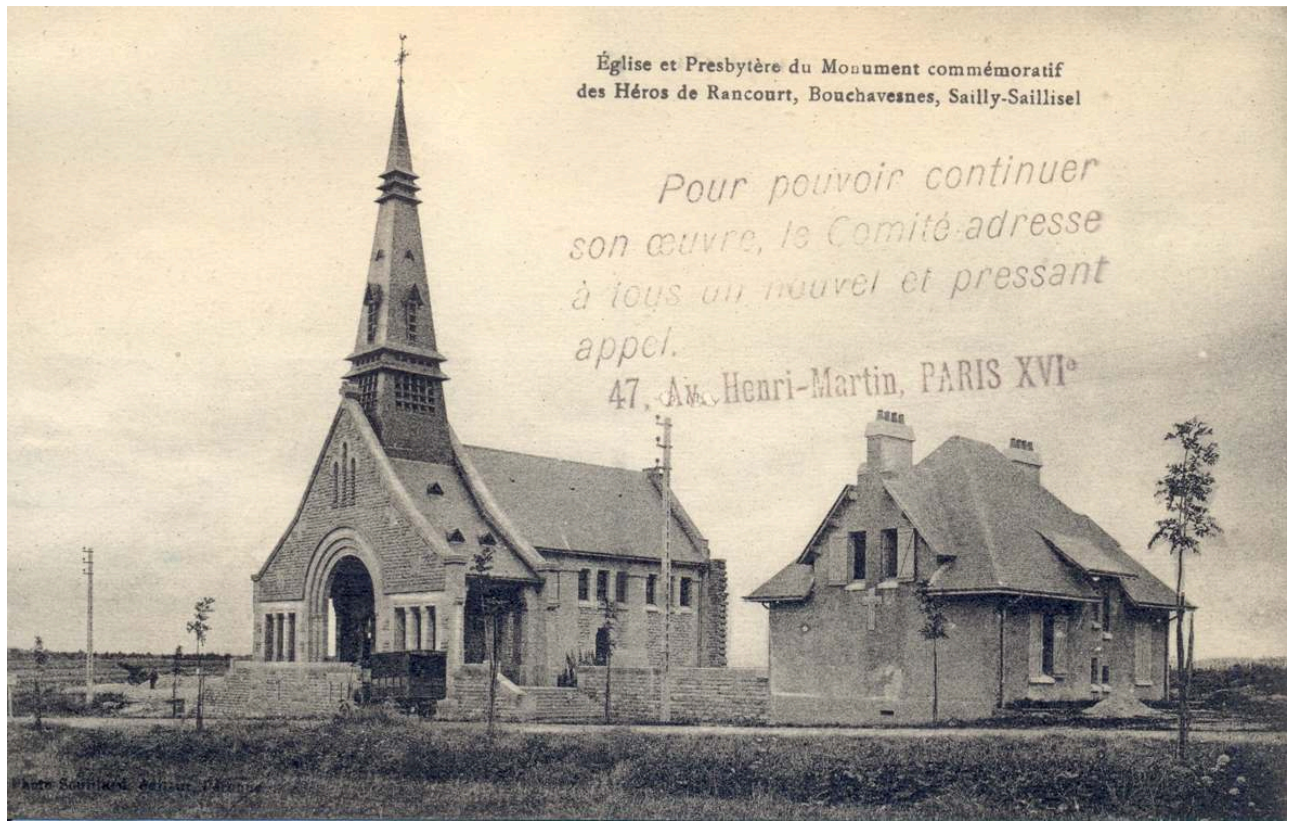

Carte postale vendue par le Comité commémoratif de la Somme représentant du monument commémoratif des Héros de Rancourt, Bouchavesnes, Sailly-Saillisse, [septembre 1926]. Archives nationales du monde du travail, fonds de la Compagnie du chemin de fer du Nord, 202 AQ 1638. Phot. Piernas, Gersende. ( ) Archives nationales du monde du travail. 
associations gravitent autour de ces pèlerinages telles l'CEuvre du retour au foyer, qui est autorisée à quêter dans les trains et à y vendre des cartes postales afin d'aider le retour des réfugiés, et autour de l'Union des grandes associations françaises contre la propagande ennemie qui y puise tous ses arguments et sa raison d'être. La question des réfugiés est importante à la fois sur place car il faut aider ceux qui sont restés mais aussi ceux qui sont partis, tels les nombreux enfants ayant été confiés à des familles parisiennes qui souhaitent aussi voir ce qui s'est produit pendant la guerre chez eux, dans le Nord. Ainsi le Comité central des réfugiés du département du Nord organise-t-il des pèlerinages pour ces enfants.

Droupes de population directement concernés par la guerre, à savoir les soldats (français, américains, anglais et canadiens), sont un temps considérés comme une clientèle potentielle. Cependant la demande, via leur ministère, de pouvoir s'y rendre en juin 1919, reçoit un accueil peu favorable de la part de la Compagnie du Nord car la démobilisation est en cours et le tarif militaire est peu rentable selon elle9

Par ailleurs, une grande partie de ces voyageurs, à travers leurs représentants (association, institution ou agence de voyage), ne se contente pas des circuits tout prêts de la Compagnie mais demande des trains spéciaux, c'est-à-dire des trains au parcours sur mesure, ce qui prouve leur intérêt et leur projet vis-à-vis des lieux visités. Toutefois, bon nombre de visiteurs, y compris ceux des circuits standard, fait savoir par correspondance à la Compagnie qu'il souhaiterait que les circuits fassent une halte plus longue à tel endroit - comme au cimetière de Lens où on déplore le comportement vandale des Allemands envers les tombes -, une autre supplémentaire à tel autre, et même, que de nouveaux tronçons soient ouverts, comme du côté du Tardenois dans l'Aisne ou du Noyonnais dans l'Oise.

41 Avec ces lettres et ces récits, on comprend que les pèlerinages se font dans une atmosphère patriotique et revancharde. Ce tourisme de mémoire et éducatif est censé répondre aux besoins de compréhension et d'interprétation des espaces de combats et des lieux de mémoire, dont le sens échapperait non seulement aux enfants mais aussi aux générations contemporaines. Les récits parvenus montrent à quel point se met en place une mythologie patriotique, guerrière et parfois mystique autour de ces sites dont la terre est devenue sacrée par le sang de ces héros. La symbolique reliquaire de ces sites, à voir, à sauvegarder comme tels, ou desquels il faut emporter un morceau, débouche inévitablement sur la volonté de commémorer le sacrifice.

42 La guerre s'approprie un espace, et la propagande anti-allemande, le pèlerinage, en font un lieu de mémoire. C'est donc à toute la Compagnie qu'en revient le mérite, quoiqu'elle puisse être fortement attaquée car, selon les habitants de la région réfugiés à Paris et qui souhaitent s'y rendre tous les jours pour reconstruire, la Compagnie donnerait priorité aux pèlerins et non à eux ${ }^{10}$.

43 Toutefois, une note interne indique que le 12 mai 1919, à son retour de pèlerinage, un agent de la Compagnie a été abordé par un homme qui souhaitait avoir quelques réponses à ses questions sur ces pèlerinages ${ }^{11}$ :

Enfin, ce dernier détail à noter : à ma sortie de la gare du Nord, j'ai été accosté par un brave homme qui m'a demandé si je ne revenais pas du train d'excursion de Lens, et m'a prié de lui fournir quelques renseignements sur la façon dont les choses s'étaient comportées : «Avait-on assez de place?", «N'allait-on pas trop vite? ", « Pouvait-on se rendre compte de l'importance des destructions? », etc. Ajoutant que sa famille tenait absolument à faire ce pèlerinage et qu'il désirait 
savoir si la chose en valait la peine. Après quelques mots de réponse, mon interviewer m'a déclaré qu'il allait de ce pas retenir ses places pour dimanche prochain.

Excursion, train de plaisir comme avant guerre, sont autant de distractions alliées au spectaculaire pour la famille. Plus que de la curiosité, il s'agit de voyeurisme qui doit être rentable au vu du déplacement opéré par toute une famille. On est bien loin des autres familles endeuillées.

Cette géographie mémorielle, basée sur toute une organisation technique et commerciale dont la Compagnie du chemin de fer du Nord est la cheville ouvrière, devient donc inévitablement une affaire touristique qui doit être rentable et qui répond aux attentes d'une partie des voyageurs.

\section{Les réalités matérielles de ces pèlerinages: vers une commercialisation de ce tourisme de mémoire, enjeu touristique}

Les témoignages des voyageurs, leurs réclamations ainsi que les notes et rapports internes de la Compagnie fournissent des éléments très précis sur la réalité des pèlerinages, bien loin de toute solennité.

Les conditions des voyages diffèrent en fonction de la nature de ces derniers. Les circuits sont assurés dans des baladeuses et combinés à des véhicules routiers alors que les trains spéciaux sont composés des wagons-lits et de wagons-restaurants. Ces baladeuses sont peu confortables avec leurs bancs en bois, leurs vitres couvertes de tôle, sans filets ni rideaux contre la pluie, ce qui est d'ailleurs rappelé dans les prospectus-horaires. Les wagons-restaurants et wagons-lits réservés aux trains spéciaux, dont le trajet est plus long, ont quant à eux « tout le confort d'un voyage au xxe siècle », selon le journaliste américain George C. Curnock en février 1919.

48 À défaut de wagons-lits et hôtels dans les villes visitées, la Compagnie installe entre juillet 1920 et septembre 1921, sur ordre du ministère des Travaux publics, dans la cour intérieure de la caserne Lévis à Arras un train-hôtel américain comme à Verdun (fig. 14). Il s'agit de 17 voitures sanitaires américaines achetées par la Compagnie française du tourisme à la liquidation des stocks à Gièvres et Saint-Thégonnec (Finistère), et à Montoir (Loire-Inférieure). L'installation est filmée par la maison Gaumont et le film est projeté la semaine du 28 août 1920 dans les salles suivantes: Aubert Palace, Lutetia, Ciné Opéra, Barbès-Palace, Brasserie Rochechouart, cinémas Lecours, Demours, Max Linder, Montcalm, Lamarck, Crystal Palace et Mozart Palace. 


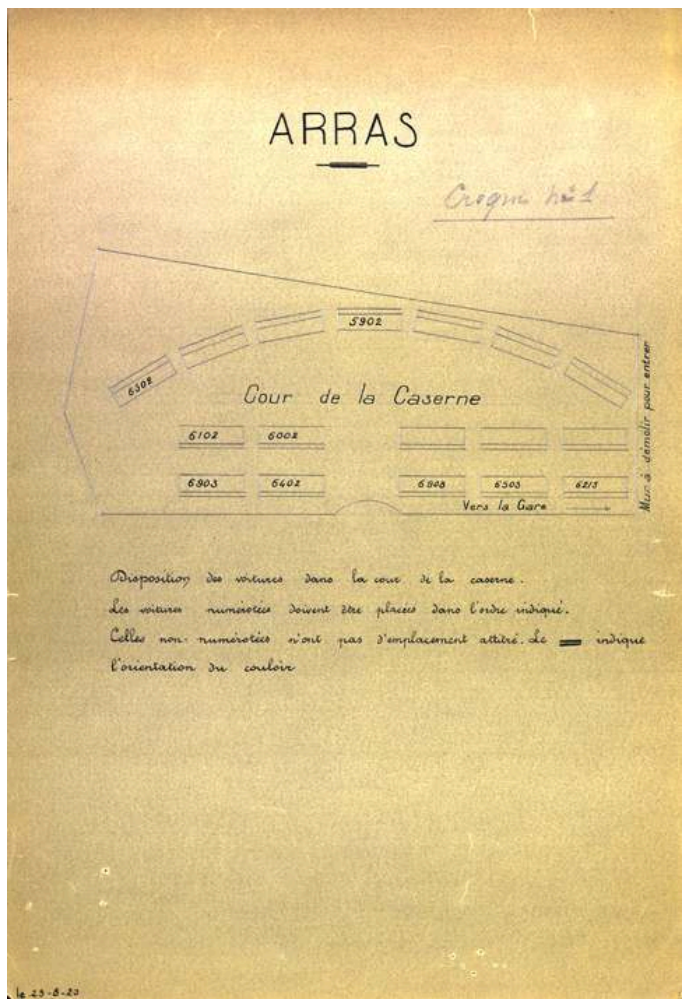

Plan au sol de l'installation des wagons-lits de l'hôtel provisoire dans la gare d'Arras, [juillet 1920]. Archives nationales du monde du travail, fonds de la Compagnie du chemin de fer du Nord, 202 AQ 1639

Phot. Piernas, Gersende. (C) Archives nationales du monde du travail.

Mais la question des repas est cruciale, en tout cas tant qu'il n'y a pas de wagonrestaurant, et pas sur la totalité du trajet. En effet, dans une note interne du 14 avril 1914, la Compagnie envisage de fournir aux voyageurs un paquet contenant « un fort pain au jambon, un morceau de fromage et un fruit $»^{12}$. Or, lors des premiers pèlerinages en mai, il n'est rien prévu à bord des premiers trains et encore moins dans les gares et les zones dévastées, comme le sous-entend le conseil de la Compagnie, qui invite dans les prospectus-horaires à emporter son repas et même à le prendre avant d'arriver à telle gare.

Lors du pèlerinage, les voyageurs se plaignent à plusieurs reprises qu'il n'y a rien à boire ni à manger à la gare d'Arras. C'est pourquoi le 14 mai 1919 la Compagnie facilite la fourniture aux voyageurs d'un panier-repas de 5,50 F (trois sandwichs importants, un fruit, une demi-bouteille de vin blanc ou rouge, un verre en carton) confectionné par la maison Duval. Elle met alors en vente des billets avec ou sans lunch.

Pourtant, dans d'autres gares avec commerces à l'intérieur et à l'extérieur comme à Soissons, les hôteliers et buffetiers disent que les voyageurs n'ont pas le temps de s'arrêter pour consommer et lancent une pétition le 25 mai 1919.

De plus, dès le mois de juillet 1919, comme les voyageurs se plaignent à Coucy-leChâteau qu'il n'y a pas de café disponible dans les gares à peine reconstruites, on autorise des cafetiers alentour à venir vendre avec des charrettes du café à la descente et à la montée des trains. 
'Ce n' qu'au mois de juin 1919 qu'il est possible de déjeuner à bord dans un wagonrestaurant, au moins au retour. Cependant, le manque d'organisation et de place ne permet pas à tous d'y accéder si bien que les réservations s'imposent rapidement (fig. 15).

Figure 15

\section{CIRCUIT COMBINÉ AVEC LES AUTO-MAILS SERVICE DU WAGON-RESTAURANT}

Afin que les places nécessaires leur soient retenues, les voyageurs désirant diner dans le wagon-restaurant du train de retour partant d'Arras à 19 h. 05 ou d'Albert à 19 h. 50 , devront se faire inscrire à leur descente du train d'aller, soit à la gare d'Albert, soit à la gare d'Arras.

Les voyageurs reprenant le train, le soir, à Arras, seront admis, au départ de cette gare dans le wagon-restaurant.

Ceux reprenant le train à Albert, seront admis dans le wagon-restaurant à partir de Longueau (20 h. 16).

Affichette pour la réservation au wagon-restaurant lors du trajet entre Arras et Albert, [juin 1920]. Archives nationales du monde du travail, fonds de la Compagnie du chemin de fer du Nord, 202 AQ 1638.

Phot. Piernas, Gersende. () Archives nationales du monde du travail.

Les rapports et les plaintes des voyageurs relatent des comportements bien éloignés du calme et du recueillement attendus. Dès les premiers circuits, les voyageurs se précipitent dans les baladeuses, se bousculent, se trompent de classe, pour lesquelles il faut instaurer rapidement un code de couleurs et un affichage clair ( $1^{\mathrm{e}}$ classe en jaune, $2^{\mathrm{e}}$ classe en vert, $3^{\mathrm{e}}$ classe en bistre) avec numérotation des voitures. Certains pèlerins jettent des bouteilles par dessus les baladeuses, d'autres quêtent ou vendent des objets sans autorisation. En outre, la demande des voyageurs d'avoir au moins deux agents de la Compagnie pour laisser leurs effets personnels dans les wagons pendant les visites indique clairement qu'il y a des risques de vol dans le train et que les voyageurs ne se sentent pas en sécurité, même entre eux.

Le pèlerinage se fait certes en commun mais il n'est pas question de se mélanger. Le désagrément des repas en commun, y compris au sein d'une même classe, dans les baladeuses, gêne. L'usage mixte des baladeuses, voiture et lieu de restauration, occasionne des salissures que ne manquent de remarquer certains voyageurs, qui demandent à la Compagnie de les faire nettoyer entre deux stations pendant les visites. Malgré tout, ces baladeuses demeurent appréciées de la majorité des voyageurs, bien 
qu'ils déplorent quelques retards lors des transbordements, plusieurs avaries et des accidents entre des voitures qui se percutent.

Le voyage et les visites sont rythmés par le son des trompettes de trois agents de la Compagnie qui donnent le signal des départs, ce qui est très apprécié de tous.

Le comportement et les réclamations des voyageurs, comme la recherche de confort par la Compagnie, font relever du tourisme les trains de pèlerinage. De surcroît, les efforts publicitaires déployés par la Compagnie montrent que ce doit être une initiative rentable.

Ces trains sont connus du grand public grâce aux prospectus-horaires, en français et traduits en anglais (fig. 16). Ils sont disponibles dans les gares, à l'office central du tourisme, dans les agences de voyage, et peuvent être envoyés par la poste sur demande en France et à l'étranger (Angleterre, Belgique, Amérique et même jusqu'en Pologne). D'autres sont distribués dans les hôtels, cercles, clubs, écoles, institutions religieuses, etc. La campagne de distribution de mai 1919 se fait avec pas moins de 50000 exemplaires.

Figure 16

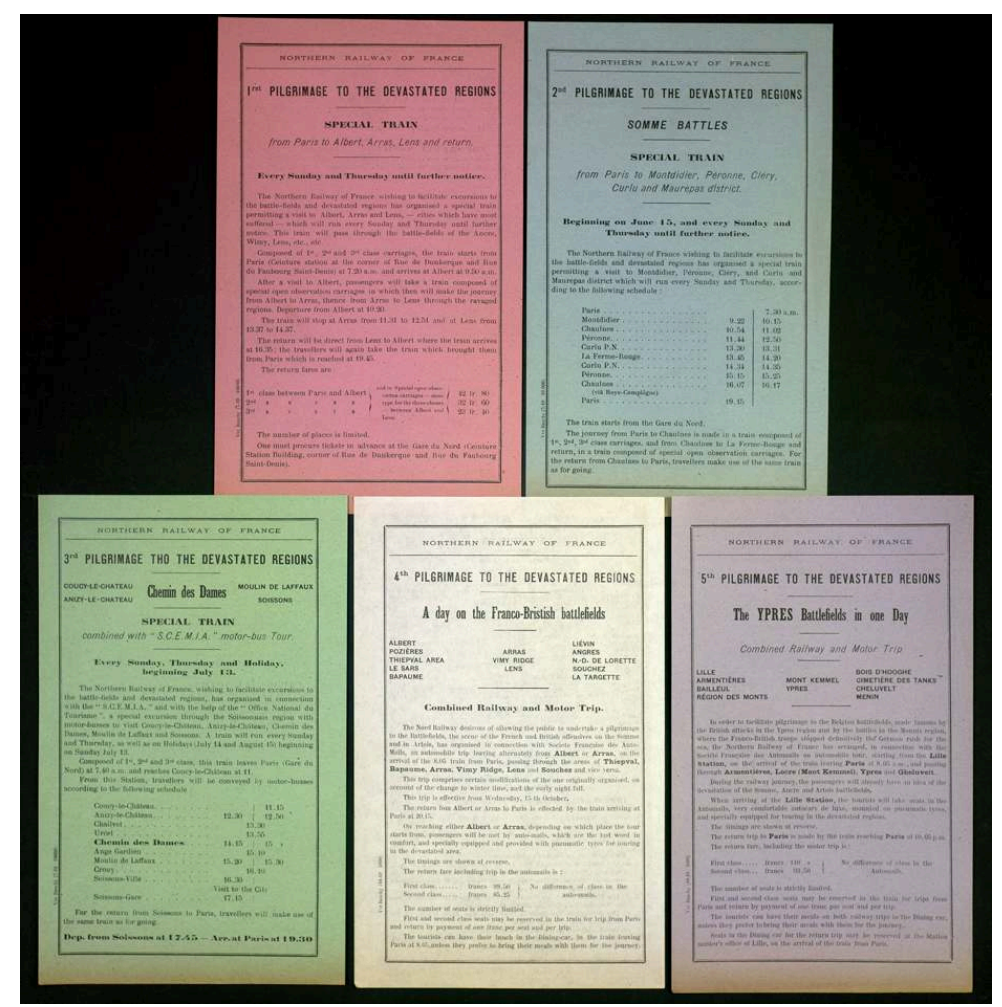

Prospectus en anglais des 5 circuits de pèlerinages de la Compagnie du chemin de fer du Nord, [1919-1920]. Archives nationales du monde du travail, fonds de la Compagnie du chemin de fer du Nord, 202 AQ 1639.

Phot. Piernas, Gersende. (c) Archives nationales du monde du travail.

Le nombre et le lieu de vente des billets de train sont aussi stratégiques. Au début, les tickets sont limités pour correspondre aux nombres de places dans les trains, soit environ 1000 , et ne sont achetables qu'à la gare du Nord (au coin de la rue de Dunkerque et rue du faubourg Saint-Denis à Paris, aux guichets 15 et 16) ou à l'agence des auto-mails, sise au 11 bis, rue Scribe à Paris. Puis les points de vente se multiplient : 
American Express, Auto-mail Agency, etc. Les billets combinés à la route sont aussi vendus dans les autres gares du réseau comme le montre la demande de la Société française des auto-mails du 28 juin 1920 pour l'installation d'une baraque de vente dans le hall de la gare d'Amiens entre les deux boutiques Hachette (fig. 17).

Figure 17

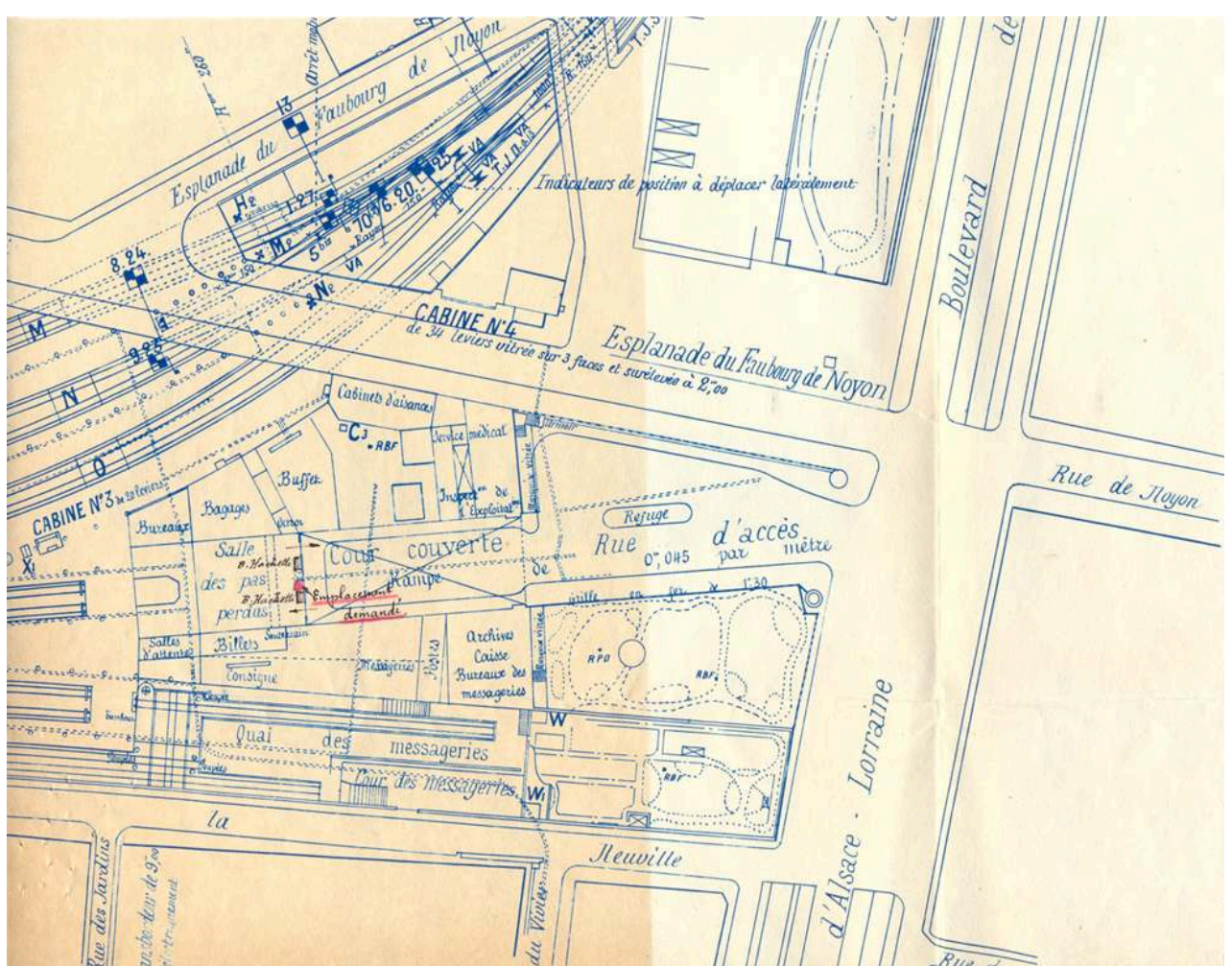

Projet d'installation d'une baraque de la Société française des auto-mails dans la gare d'Amiens 28 juin 1920. Archives nationales du monde du travail, fonds de la Compagnie du chemin de fer du Nord, 202 AQ 1639.

Phot. Piernas, Gersende. (C) Archives nationales du monde du travail.

La diffusion de ces prospectus et la vente des billets sont largement appuyées par une importante campagne d'affiches. Plusieurs séries sont demandées à des artistes, souvent de jeunes étudiants des Beaux-Arts qui cèdent leurs droits à la Compagnie, mais aussi des affichistes confirmés, Charles-Jean Hallo, dit ALO (1882-1969), Henri Gray (1858-1924), Julien Lacaze (1886-1971), entre autres.

61 Il s'agit de dessins de paysages urbains ou de champs de bataille dévastés dupliqués dans le service des imprimés de la gare du Nord. Ces lithographies ont des coloris simples afin de donner une impression forte de la dévastation et d'attirer l'attention. Ces affiches sont donc bien éloignées de celles d'avant guerre réalisées parfois par les mêmes artistes et qui représentaient des sites, des villes et des paysages très vivants et très colorés.

Ces affiches en français ou en anglais sont envoyées régulièrement aux partenaires (SCEMIA et Auto-mails), aux autres compagnies de chemin de fer françaises, belges et anglaises, à l'Église de France, aux grandes banques, aux agences de voyages et ce afin d'être apposées dans leurs locaux respectifs, à Paris comme en province. Il en subsiste quelques-unes dans le fonds de la Compagnie (fig. 18 à 22). 
Figure 18

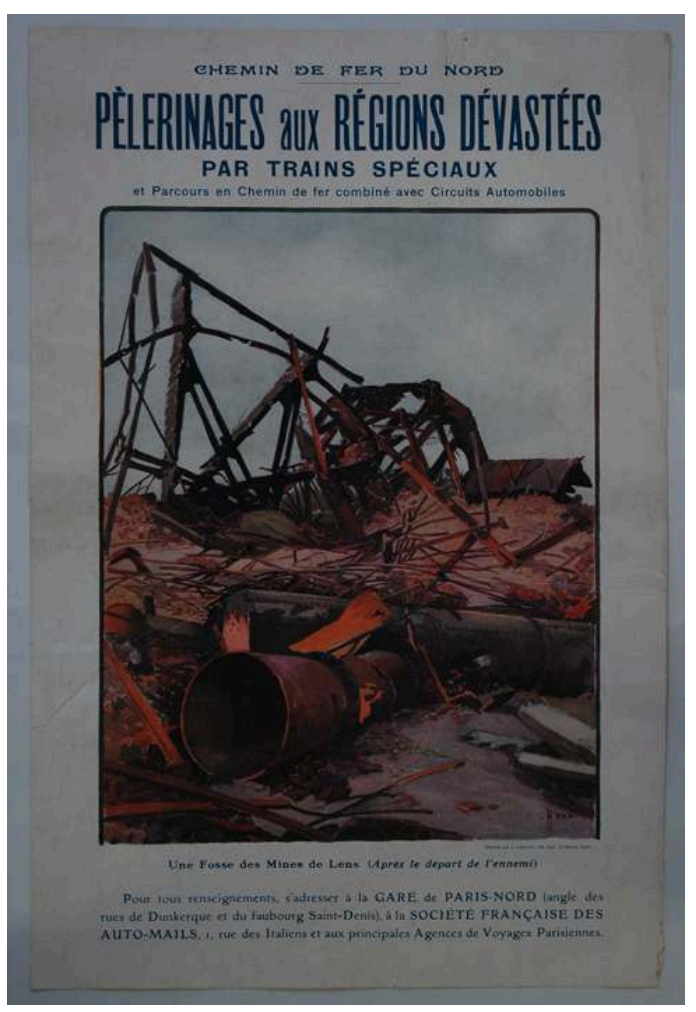

Affiche publicitaire de la Compagnie du chemin de fer du Nord, «Pèlerinages aux régions dévastées, par trains spéciaux » [une fosse des mines de Lens], Imprimerie Langlois, Paris, $59 \times 39 \mathrm{~cm}$, affiche non entoilée, [1919-1920]. Archives nationales du monde du travail, fonds de la Compagnie du chemin de fer du Nord, 202 AQ 1893.

Phot. Piernas, Gersende. ( ) Archives nationales du monde du travail. 
Figure 19

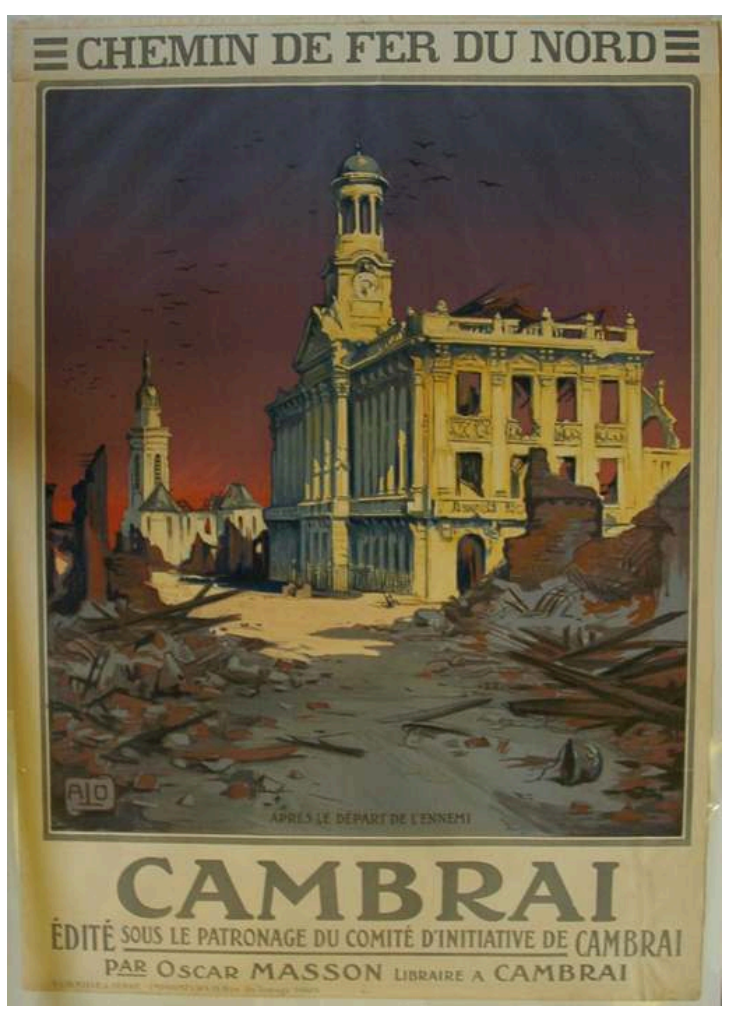

Affiche publicitaire de la Compagnie du chemin de fer du Nord, « Cambrai, après le départ de l'ennemi ", ALO (Charles-Jean Hallo), imprimerie Cornille \& Serre, Paris, $104 \times 75$ cm, non entoilée, [1919-1920]. Archives nationales du monde du travail, fonds de la Compagnie du chemin de fer du Nord, 202 AQ 1896.

Phot. Piernas, Gersende. (C) Archives nationales du monde du travail. 
Figure 20

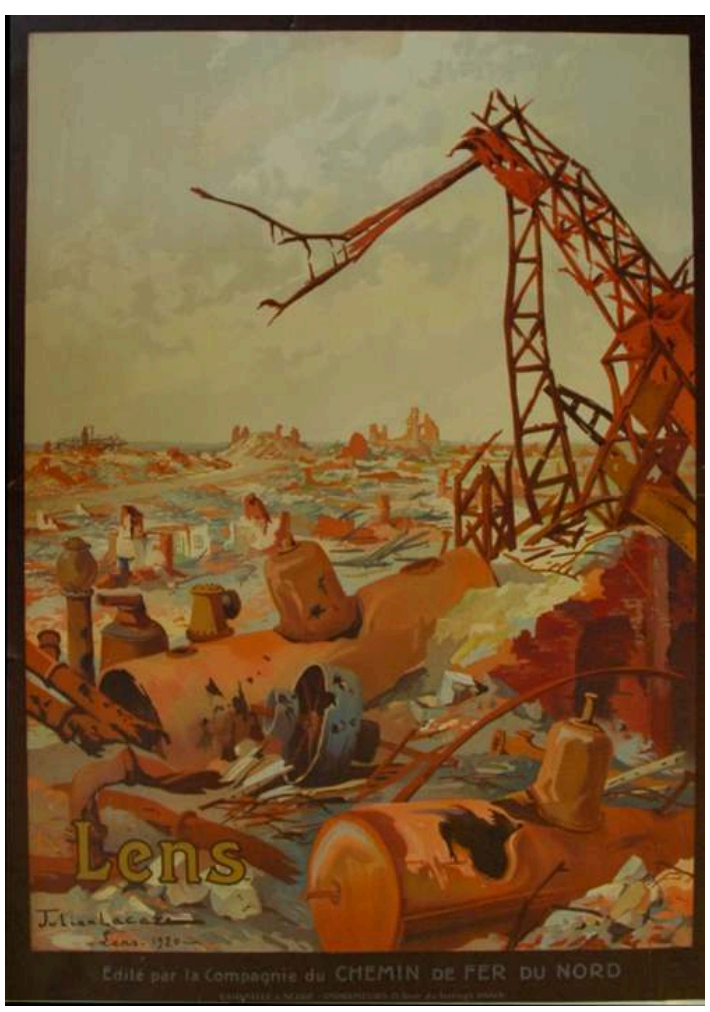

Affiche publicitaire de la Compagnie du chemin de fer du Nord, «Lens, 1920 ». Julien Lacaze, Imprimerie Cornille \& Serre, Paris, $104 \times 75 \mathrm{~cm}$, non entoilée, [1920]. Archives nationales du monde du travail, fonds de la Compagnie du chemin de fer du Nord, 202 AQ 1892.

Phot. Piernas, Gersende. (C) Archives nationales du monde du travail. 
Figure 21

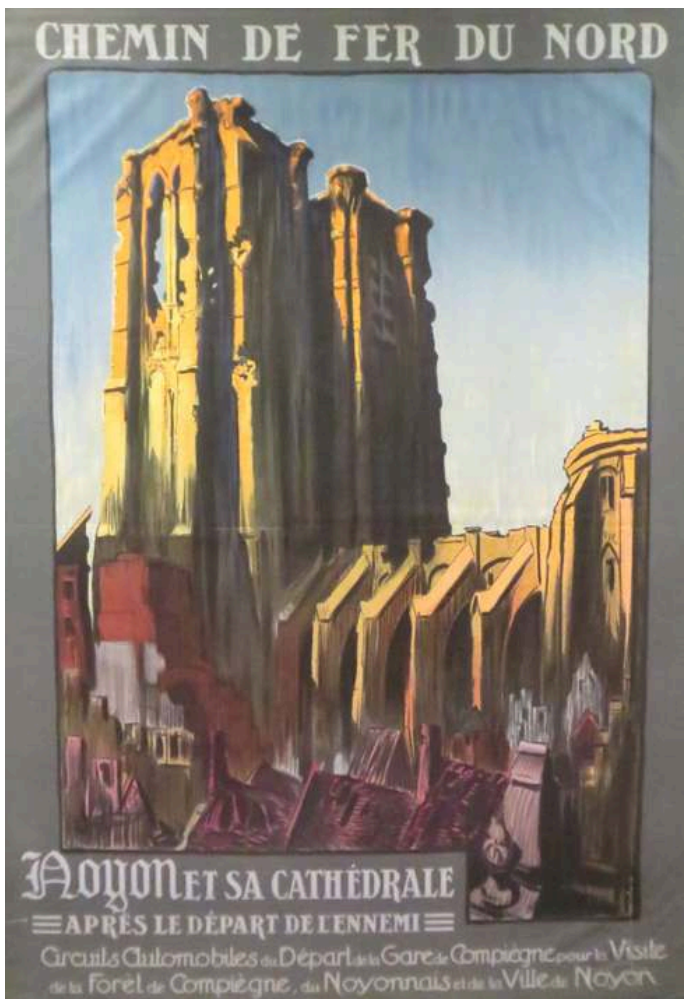

Affiche publicitaire de la Compagnie du chemin de fer du Nord, « Noyon sa cathédrale après le départ de l'ennemi ». [Joe Fourmi ?], imprimerie G. Jenin, Paris, $104 \times 74$ cm, non entoilée, [1920]. Archives nationales du monde du travail, fonds de la Compagnie du chemin de fer du Nord, 202 AQ 1895.

Phot. Piernas, Gersende. (c) Archives nationales du monde du travail. 
Figure 22

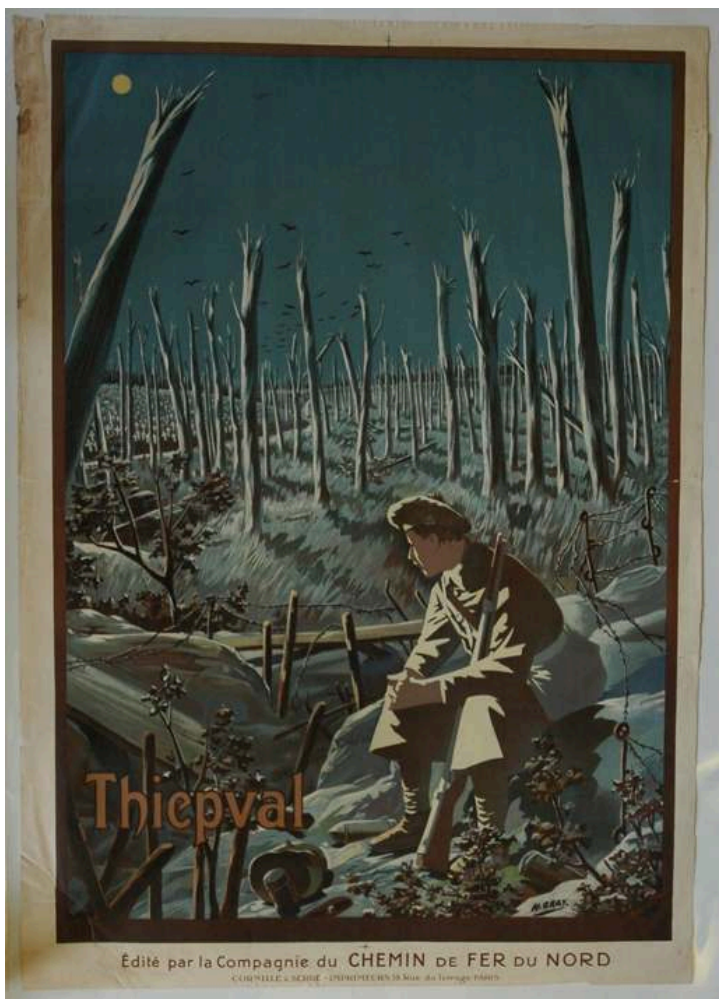

Affiche publicitaire de la Compagnie du chemin de fer du Nord, « Thiepval » [paysage dévasté, soldat assis au sol, fusil posé, cimetière]. Henri Gray, Imprimerie Cornille \& Serre, Paris, 104 x $75 \mathrm{~cm}$, non entoilée, [1920]. Archives nationales du monde du travail, fonds de la Compagnie du chemin de fer du Nord, 202 AQ 1894.

Phot. Piernas, Gersende. (C) Archives nationales du monde du travail.

Parmi toutes les séries, tirées à plus de 30000 exemplaires en 1920, celle qui semble avoir le plus de succès d'après les nombreuses demandes d'envois est celle de Julien Lacaze représentant les destructions de la fosse de Lens.

Leur affichage en gare est également stratégique. C'est pourquoi le chef d'exploitation veille à leur bon emplacement à l'intérieur et à l'extérieur de la gare du Nord dans une lettre du 23 mai 1919 adressée à l'inspecteur chef des services :

«J'ai constaté que les affiches spéciales du train de pèlerinage Albert-Arras-Lens, n'étaient pas affichées, en gare de Paris, en des points tels que le public qui circule dans la gare du Nord ou à des alentours ait nécessairement les yeux attirés par ces affiches".

«Je vous prierai de vouloir bien faire placer des affiches dans la gare de Paris, bien en vue, en des endroits parfaitement éclairés. Voulez-vous même examiner la possibilité d'en mettre un certain nombre à l'extérieur, sur la façade principale, et à une certaine hauteur au-dessus du sol, de façon à éviter qu'elles soient lacérées ${ }^{13}$ ”.

À côté de cette campagne d'affichage, la Compagnie du Nord réalise plusieurs dioramas comme celui intitulé Des chemins des Dames ${ }^{14}$. Elle envoie aussi de nombreuses notes à la presse quotidienne française, anglaise, américaine et belge afin d'y publier des encarts publicitaires dans les petites informations. Ces insertions sont très courtes comme celle préparée le 26 avril 1919 pour le circuit Arras-Albert-Lens :

Le parcours ayant lieu dans des voitures baladeuses, couvertes seulement d'un toit parapluie, M.M. Les Voyageurs sont expressément invités à se vêtir en conséquence. 
Ils devront emporter avec eux leur déjeuner. Il sera difficile d'acheter à Arras autre chose que de la boisson ${ }^{15}$.

Parallèlement, la Compagnie se rend aussi disponible à la presse pour tous les articles qu'elle rédigeait sur ces pèlerinages, n'hésitant pas à fournir des photographies directement.

La publicité passe aussi par des partenariats ou des ententes avec les compagnies de chemin de fer, d'automobiles ou autocars, mais aussi des compagnies de navigation. Ainsi, début novembre 1919, la Société française des auto-mails informe la Compagnie $\mathrm{du}$ Nord qu'elle souhaite se mettre en rapport avec les Compagnies de chemin de fer anglaises et de navigation et les grandes entreprises de tourisme anglais pour accueillir au printemps 1920 des touristes anglais sur les champs de bataille de la région anglaise $\mathrm{du}$ front. Des rapprochements sont également opérés avec la Compagnie générale transatlantique afin d'obtenir plus de pèlerins en provenance d'Amérique du Nord.

En plus de la vente des billets, la Compagnie prévoit la vente d'articles souvenirs. Dans une note interne du 14 avril 1914, la Compagnie envisage de vendre aux voyageurs avec le billet un bidon et un quart sur lesquels serait frappé «Souvenir du Pèlerinage à Albert-Arras-Lens dévastés ${ }^{16}$. Des guides et des brochures de visite en français et en anglais pour chaque circuit sont édités et mis en vente à $2 \mathrm{~F}$ par la Compagnie dans les gares, par l'intermédiaire de leur réseau des librairies Hachette mais aussi dans les trains eux-mêmes (fig. 23) alors que les concurrents les mettent gracieusement à la disposition des voyageurs. Ces guides, véritables instruments de convalescence psychique, aides au travail de deuil à travers des descriptifs militaires, des photographies et des points de vue, sont vendus à plus de 10000 exemplaires en France et 30000 en Angleterre. 
Figure 23

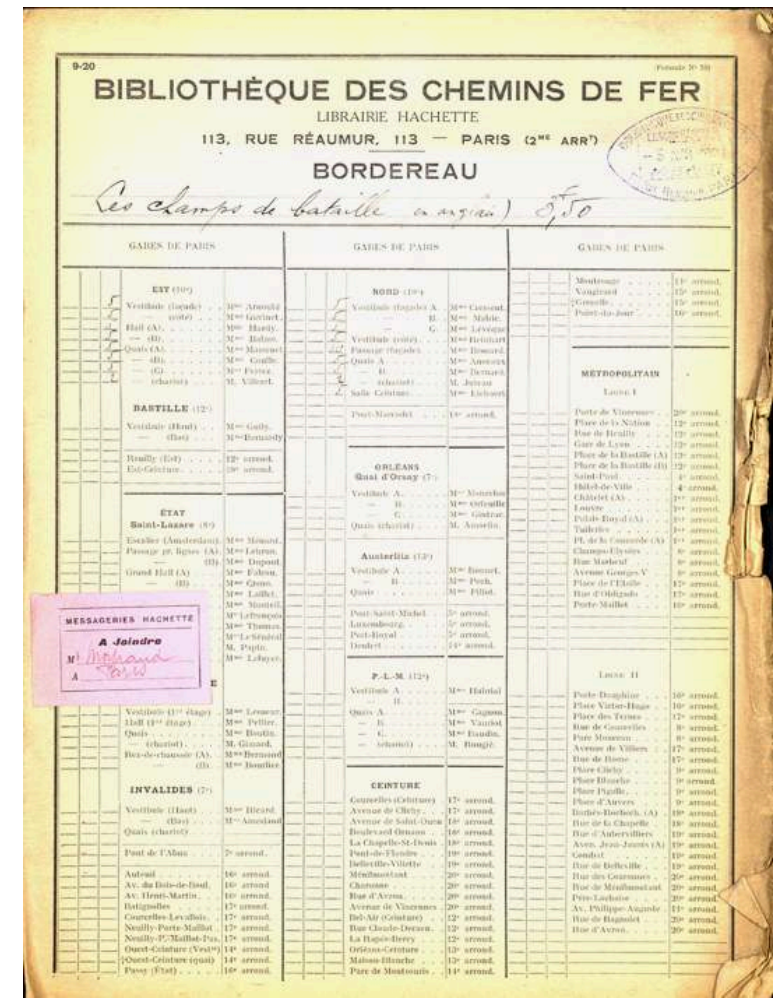

Liste du nombre d'exemplaires du guide Les champs de bataille édité par la Compagnie du chemin de fer du Nord et vendu par les librairies de gare Hachette, 5 avril 1921. Archives nationales du monde du travail, fonds de la Compagnie du chemin de fer du Nord, 202 AQ 1639.

Phot. Piernas, Gersende. (C) Archives nationales du monde du travail. trains des ouvrages d'auteurs en lien avec les régions dévastées, comme celui d'Henri Bidou, La terre héroïque, publiée en 1919 à Paris à l'Imprimerie de Vaugirard.

Elle fait aussi vendre dès 1919 par les conducteurs-guides des cartes postales à l'unité ou en bloc dans les trains puis en 1920 des phototypies représentant la basilique d'Albert, l'hôtel de ville d'Arras et la place de l'église de Lens. Ces phototypies sont proposées par l'Union phototypique parisienne qui en vend avec des panoramas de Verdun à Verdun même et à Paris. Selon une note de la Compagnie du Nord du 19 mai 1920, cette dernière accepte ces ventes car :

ces vues présentent actuellement un intérêt documentaire, principalement la vue de la place de Lens, car le tumulus de l'Église, aperçu sur la vue photographique, est maintenant déblayé et les pèlerins pourront également faire la comparaisons des travaux énormes de déblaiement effectués dans la ville de Lens ${ }^{17}$.

71 L'intérêt est certes historique mais très vite dans le déroulement de cette note, la Compagnie calcule que les 2000 reproductions en noir et blanc seraient bien moins chères que celles en couleurs.

Tous les articles vendus à bord des trains par les conducteurs-guides leur procurent un petit bénéfice de $10 \%$ par unité.

73 Ces publicités, ces ventes d'objets et cette recherche de profits sont cependant nécessaires à la Compagnie afin que ces opérations soient rentables et qu'elle puisse faire face à la concurrence des entreprises automobiles et des régions belges dévastées. 
Figure 24

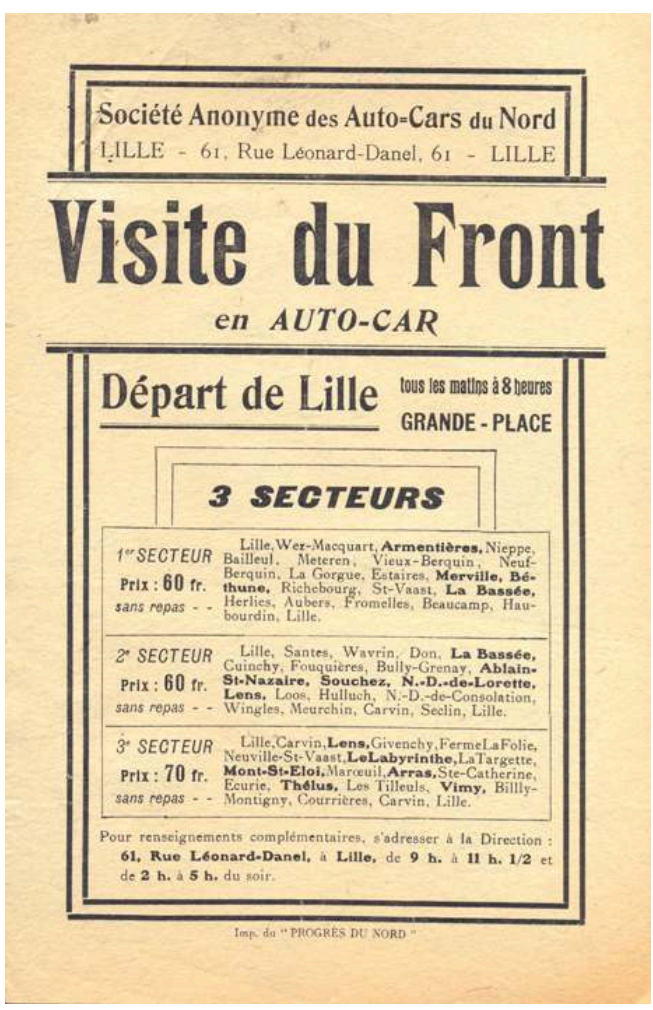

Prospectus de la Société anonyme des autocars du Nord pour des visites au front en autocars à partir de Lille, s.d. Archives nationales du monde du travail, fonds de la Compagnie du chemin de fer du Nord, 202 AQ 1638.

Phot. Piernas, Gersende. (c) Archives nationales du monde du travail.

En effet, la mise en place des pèlerinages dans les régions dévastées n'est pas que l'apanage de la Compagnie du chemin de fer du Nord et de celle de l'Est mais aussi le fait de nombreuses entreprises de transport par automobiles et autocars, autres que celles associées à ces deux compagnies (fig. 24). Ces initiatives privées, lucratives, exploitent sans scrupules le filon, si bien que les pouvoirs publics tentent de moraliser la situation. Ainsi, le 11 mars 1920, une proposition de loi tend à frapper d'une taxe spéciale les entreprises de toute sorte qui ont pour objet la visite des champs de bataille, soit un impôt de 250/0 sur le bénéfice net des entreprises privées et publiques ${ }^{18}$. Cette taxe serait au profit des mutilés, veuves et orphelins de guerre. Ceci dit, le long préambule de cette proposition laisse clairement penser que les autorités entérinent ces initiatives qui sont nécessaires et édicte la sacralité de ces lieux :

Les champs de bataille de France, où s'est déroulée la lutte épique de la civilisation contre la force brutale, doivent être librement ouverts au monde entier. Les peuples de l'univers doivent pouvoir venir y contempler, sans contrainte, les lieux qui furent l'objet et le témoin de si nobles héroïsmes et de si grands sacrifices, et y prendre des leçons de désintéressement et d'abnégation. Nous devons pouvoir y aller nous-mêmes pleurer nos morts, et vivre quelques instants de cette atmosphère de grandeur qui s'y dégage de l'âme des choses. Ces lieux sacrés doivent rester une page, ouverte à tous, de notre histoire nationale.

Malgré ce projet, la dérive touristique est inévitable, y compris à la Compagnie du Nord: profits de la compagnie, partenariats et concurrence à gérer. Ces pèlerinages 
deviennent un enjeu touristique pour la Compagnie, ce qui est largement encouragé par les intentions et le comportement des pèlerins eux-mêmes.

Ainsi, les trains de pèlerinage de la Compagnie du chemin de fer du Nord, opération initiée par le ministère de la Guerre, sont un succès sur la courte période pour laquelle nous avons des informations complètes. Il montre la capacité de cette compagnie à se relever et à être un vecteur utile face à ce traumatisme national. Les circuits standard ou à la demande permettent de sillonner une région dévastée qui devient vite un espace mémoriel national. Ils donnent à voir les dégâts au sein du réseau Nord mais aussi des campagnes, des usines et des villes. Ils donnent ainsi accès à ce patrimoine détruit dont il faut témoigner de l'état pour démontrer la barbarie ennemie mais dont il faut aussi estimer les frais de reconstitution (reconstruction, remise en état ?: le terme de reconstitution est bien celui employé à l'époque car il s'agit de reconstruire à l'identique). L'engouement des nombreux visiteurs français et étrangers et leur volonté de conserver des débris en souvenir montrent à quel point la Grande Guerre, à peine terminée, fascine tant par les pertes humaines sans précédent que par les destructions et leur étendue. Ces premiers témoins civils, témoignant à leur tour, génèrent alors un imaginaire collectif, à partir des champs de bataille et au sujet de l'héroïsme des soldats, qui prend la forme au niveau officiel de monuments et mémoriaux, cimetières et nécropoles et de commémorations autour de la date du 11 novembre. Néanmoins, à travers leur comportement parfois décalé et les actions de la Compagnie du Nord, qui demeure une entreprise proposant des circuits avant tout attractifs et rémunérateurs, le tourisme avec tous ses à-côtés est sous-jacent. Les campagnes de publicité et de marketing sont poussées à l'extrême mais n'ont pas raison de la perte d'audience, liée à l'éloignement de la fin du conflit tout comme à la concurrence croissante de la route.

Cette découverte des régions dévastées s'estompe pour renaître pleinement sous la forme de redécouverte, bien plus tard, notamment à l'occasion des grands anniversaires et des enjeux européens de réconciliation à la fin $\mathrm{du} \mathrm{xx}^{\mathrm{e}}$ siècle. C'est ainsi qu'au moment du décès du dernier poilu français, Lazare Ponticelli ${ }^{19}$, en 2008 , les Archives nationales du monde du travail décidèrent de lui rendre hommage, à lui et à l'ensemble des combattants de la Grande Guerre, en sélectionnant une dizaine de documents issus de ces archives de la Compagnie du chemin de fer du Nord, pour en faire une lecture le 18 avril 2008 devant le public du Cabaret de l'Union ${ }^{20}$.

Cet article montre donc que ces archives sont une preuve de l'histoire du patrimoine dans la guerre, comme de la naissance du patrimoine de la Grande Guerre, et ce avant même la fin du conflit, devenant elles-mêmes une forme de ce patrimoine.

\section{NOTES}

1. - Archives nationales du monde du travail, fonds de la Compagnie du chemin de fer du Nord, 202 AQ 1638, note des services techniques à l'ingénieur en chef de l'exploitation du 4 avril 1919 et état des travaux de reconstitution des ouvrages d'art du $1^{\mathrm{er}}$ juillet 1920. 
2. - Ibid. $202 \mathrm{AQ} 1638-1639$, dossier sur les trains de pèlerinages organisés dans les régions dévastées du Nord, 1919-1926.

3. - Ibid. 202 AQ 401, compte rendu d'une réunion à l'ONT, $1^{\mathrm{er}}$ mars 1918.

4. - Voitures confortables montées sur pneumatiques et spécialement aménagées pour la visite des régions dévastées.

5. - BOUTIN, Ludovic. Chemin de fer \& tourisme. La Compagnie du chemin de fer du Nord, 1846-1937. Mémoire de maîtrise en histoire contemporaine sous la direction d'Emmanuel Chadeau, Université Charles de Gaulle-Lille III, juin 1997, p. 53-67.

6. - ANMT, fonds de la Compagnie du chemin de fer du Nord; voir l'article de : OWEN KUHN, Olivier. Après une visite au Nord de la France. L'indignation contre le peuple allemand d'un journaliste américain. s.l.n.d. [26 février 1919], 202 AQ 1638.

7. - Les réductions et exonérations demandées à la Compagnie ne sont pas toutes accordées, et lorsqu'elles le sont, les critères d'attribution manquent de transparence voire d'équité. Afin de les obtenir, certains n'hésitent pas à citer les morts pour la France de leur entourage.

8. - ANMT, fonds de la Compagnie du chemin de fer du Nord, demande de réduction par la Caisse des écoles de Montreuil le 26 juillet 1919, 202 AQ 1638.

9. - Ibid. Note interne du 9 juillet 1919.

10. - Ibid. Article «Les Pèlerins passent avant les sinistrés» dans Le Pas-de-Calais Libéré, 26 juin 1919.

11. - Ibid. Note interne du 12 mai 1919

12. - ANMT, fonds de la Compagnie du chemin de fer du Nord, 202 AQ 1639.

13. - ANMT, fonds de la Compagnie du chemin de fer du Nord, $202 \mathrm{AQ} 1638$

14. - ANMT, fonds de la Compagnie du chemin de fer du Nord, 202 AQ 399, dépenses des publicités touristiques de 1920 à 1923.

15. - ANMT, fonds de la Compagnie du chemin de fer du Nord, 202 AQ 1638.

16. - ANMT, fonds de la Compagnie du chemin de fer du Nord, 202 AQ 1639.

17. - Ibid

18. - ANMT, fonds de la Compagnie du chemin de fer du Nord, 202 AQ 1638

19. - Lazare Ponticelli, né Lazzaro Ponticelli le 24 décembre 1897 à Bettola en Italie et mort le 12 mars 2008 au Kremlin-Bicêtre en France, est officiellement le dernier vétéran français de la Première Guerre mondiale.

20. - Cette lecture d'archives, intitulée «Le mythe d'une commémoration. 14-18: les champs de bataille de la Somme ", avait été préparée et lue par l'auteur. Le Cabaret de l'Union était une manifestation culturelle mensuelle organisée de 2006 à 2010 par les Archives nationales du monde du travail et l'association TEC-CRIAC, à l'initiative de Françoise Bosman, directrice des ANMT. Ce cabaret avait lieu 10 fois par an, le troisième vendredi du mois aux ANMT, dans un espace dédié et muni d'une scène et d'un mobilier spécifique (tables, chaises, fauteuil), réalisé par l'artiste Bernard Agnias à partir de matériaux de récupération d'usines textiles. Le thème de la soirée était choisi en commun par les ANMT et TEC-CRIAC, et était décliné en lecture de documents, projection audiovisuelle, débat et performances artistiques. 


\section{ABSTRACTS}

As early as 1919, the Compagnie du chemin de fer du Nord, the North railway company, organised regular circuits to visit the French and British battlefields of the First World War, departing from Paris or other large towns in the north. These circuits, with aspects that were political, economic, tourist and historic, were described as 'pilgrimages', to counter the propaganda of the now defeated enemy which had denied the reality of its destructions. The organisation of these trips demonstrated a will to share the history of the recent conflict with a large public, contributing to the development of myths surrounding the war, its protagonists such as the 'Poilu', the French infantryman, and the debt to the allied forces of liberation.

La Compagnie du chemin de fer du Nord met en place dès 1919 des circuits réguliers pour visiter les champs de bataille franco-anglais, à partir de Paris et de certaines villes du Nord. Entre enjeux politiques, économiques, touristiques et historiques, ces circuits appelés « pèlerinages » mettent en lumière la nécessité de contrer la propagande de l'ancien ennemi, niant les destructions, et révèlent la volonté de faire connaître au public un conflit tout proche, ce qui contribue à la naissance du mythe de la Grande Guerre, du Poilu et à la reconnaissance envers les Alliés libérateurs.

\section{INDEX}

Keywords: posters, coach, battlefield, railway, cemetery, tourist guide, ruined monuments, press, publicity, soldier, souvenir, tourism, pilgrimage train, ruined city ; allies, Aisne, Nord, Oise, Somme, America, England, Belgium, Canada, the Netherlands, the Battle of the Somme, the Chemin des Dames, peace conference

Mots-clés: affichage, autocar, champ de bataille, chemin de fer, cimetière, guide touristique, monument en ruine, presse, publicité, réfugié, soldat, souvenir, tourisme, train de pèlerinage, ville en ruines, Alliés, Aisne, Nord, Oise, Somme, Amérique, Angleterre, Belgique, Canada, PaysBas, Bataille de la Somme, Chemin des Dames, Conférence pour la paix

\section{AUTHOR}

\section{GERSENDE PIERNAS}

archiviste, chargée d'études documentaires, responsable du Pôle Archives d'entreprises aux Archives nationales du monde du travail gersende.piernas@culture.gouv.fr 